\title{
A Mini Review on Nanocarbon-Based 1D Macroscopic Fibers: Assembly Strategies and Mechanical Properties
}

\author{
Liang Kou ${ }^{1}$ Yingjun Liu $^{2} \cdot$ Cheng Zhang ${ }^{1} \cdot$ Le Shao $^{1} \cdot$ Zhanyuan Tian $^{1} \cdot$ Zengshe Deng $^{1} \cdot$ Chao Gao $^{2,3}$
}

Received: 9 June 2017/Accepted: 11 July 2017/Published online: 16 August 2017

(C) The Author(s) 2017. This article is an open access publication

\begin{abstract}
Nanocarbon-based materials, such as carbon nanotubes (CNTs) and graphene have been attached much attention by scientific and industrial community. As two representative nanocarbon materials, one-dimensional CNTs and twodimensional graphene both possess remarkable mechanical properties. In the past years, a large amount of work have been done by using CNTs or graphene as building blocks for constructing novel, macroscopic, mechanically strong fibrous materials. In this review, we summarize the assembly approaches of CNT-based fibers and graphene-based fibers in chronological order, respectively. The mechanical performances of these fibrous materials are compared, and the critical influences on the mechanical properties are discussed. Personal perspectives on the fabrication methods of CNT- and graphene-based fibers are further presented.
\end{abstract}

Keywords One dimensional · Macroscopic architectures · Carbon nanotubes · Graphene fibers · Assembly strategies · Mechanical performance

\section{Introduction}

Nanocarbon-based materials, such as carbon nanotubes (CNTs) and graphene, are extensively investigated due to their outstanding properties such as large theoretical specific area, high Young's modulus $(E)$, unparalleled

Liang kou and Yingjun Liu have contributed equally to this work.

Liang Kou

kouliang@sxccti.com

$\triangle$ Chao Gao

chaogao@zju.edu.cn

1 Shaanxi Coal and Chemical Technology Institute Co., Ltd, 2 Jinye Road 1, Xi' an 710070, People's Republic of China

2 MOE Key Laboratory of Macromolecular Synthesis and Functionalization, Department of Polymer Science and Engineering, Zhejiang University, Hangzhou 310027, People's Republic of China

3 State Key Laboratory for Modification of Chemical Fibers and Polymer Materials, Donghua University, Shanghai 201620, People's Republic of China thermal and electricity conductivity, and ultrahigh electron mobilities [1-4]. It is of practical significance to integrate the superior physical and chemical properties of individual CNTs or graphene to the macroscopic level by assembling them into macroscopic architectures [4]. As a representative of high-performance fibers, carbon fibers featured by their high tensile strength $(\sigma)$ and $E$ are widely used in aerospace, aviation, automobile, electronic, mechanical, chemical, textile, and other civilian industry. However, the electrical and thermal conductivities of carbon fibers are not satisfied. One-dimensional (1D) macroscopic architecture of CNTs and graphene, namely CNTs fibers (CFs) and graphene fibers (GFs), are analogous to carbon fibers in constitution and structure. Moreover, the combined performances including good flexibility and excellent electrical and thermal conductivities make them potential as structural-functional integrated materials. As more attentions have been attracted to the macroscopic fibers of $1 \mathrm{D}$ CNTs and 2D (two-dimensional) graphene, it is the time to give a correlative review about CFs and GFs together. We think that this insightful review can help researchers to have a better systematic understanding of the whole 
developing journey of macroscopic assembled fibers. In this mini review, we firstly introduce the assembly strategies of CFs and GFs and then compare the mechanical performance of fibers made from different approaches. Finally, we present our perspectives on the future of CFs and GFs.

\section{CNTs Fibers: 1D CNTs Macroscopic Architectures}

Macroscopic fibers containing only CNTs will yield great advances in high-tech applications (e.g., aerocraft, lightweight cables) if they can attain the extraordinary mechanical and electrical properties of individual CNTs. This requires that the CNTs in the fibers are sufficiently long, highly aligned, and packed in an arrangement, and is nearly free of defects. Until now, the strategies for assembling CNTs to CFs can be classified into liquid- or solid-state spinning approaches. Liquid-state spinning approaches involve the dispersing of CNTs in a suitable solvent and shaping in coagulation bath. Solid-state spinning approach circumvents the dissolution problem either by drawing a fiber from a vertically grown CNT array or by drawing directly from an aerogel in the furnace [5].

\subsection{Liquid-State Spinning Approaches}

Liquid-state spinning approaches generally include polymer-based coagulation spinning method, liquid crystalline (LC) spinning method, polymer-free spinning method, and dip-coating method. Among them, polymer-based coagulation spinning method is first introduced to achieve CFs.

\subsubsection{Polymer-Based Coagulation Spinning Method}

The history of CFs fabrication dates back as far as 17 years ago. In 2000, Poulin's group dispersed single-wall carbon nanotubes (SWNTs) in sodium dodecyl sulfonate (SDS) solutions, recondensed the nanotubes in the flow of poly(vinyl alcohol) (PVA) solution to form a nanotube mesh, and then collated this mesh to a nanotube fiber (Fig. 1a-c). When SWNTs dispersion was slowly injected through a syringe needle or a thin glass capillary, flow-induced alignment of the nanotubes occurred in the direction of the fluid velocity. The flow-induced alignment was maintained by the PVA solution since it allows the SWNTs to be rapidly stuck together. The as-made CNT/PVA fibers possess good mechanical performance with $150 \mathrm{MPa}$ for $\sigma$ and $15 \mathrm{GPa}$ for $E$ [6]. Replaced SDS surfactant with DNA, the CFs were also mechanically strong and conductive [7]. Scanning electron microscopy (SEM) images reveal that the fiber has a circular cross section with $80 \mu \mathrm{m}$ in diameter (Fig. 1d) and well-resolved network of DNA-SWNT hybrid (Fig. 1e) [8].

By utilizing SWNTs synthesized from carbon monoxide and lithium dodecyl sulfate as a surfactant, Baughman's groups first produced mechanically strong gel fibers. After post-treatment with acetone-washing bath, continuous, multifunctional dry CFs were achieved. The resultant CFs showed a dramatically increased $\sigma$ and toughness (570 $\mathrm{J} \mathrm{g}^{-1}$ ) [9, 10] Besides, hot-drawing technique was utilized to improve the properties of CFs [11]. The resulting 10-m-long fiber collected onto a small winder is shown in Fig. 1f. This treatment induced a crystallinity increase in the PVA and an unprecedented degree of alignment of the CNTs, leading to a markedly improved energy absorption at low strain [11].

\subsubsection{LC Spinning Method}

The main challenge of producing CFs is to disperse the CNTs at high concentration for efficient alignment. Using surfactant is an effective approach to disperse CNTs into solutions, yet there is a loss in the properties of CFs due to the decreased van der Waals interaction between the neighboring CNTs. So super-acid (100\% sulfuric acid) was introduced to increase the dispersity of CNTs. The protonated SWNTs in the superacid behave as rigid-rod-like liquid crystals (LC) and exhibit an isotropic-to-nematic phase transition with an increased concentration (Fig. 2a, b) [12-14]. Based on this behavior, LC spinning technique was proposed to produce highly orientated CF $[15,16]$. Windle's group spun fibers consisting of multi-wall carbon nanotubes (MWNTs) directly from the lyotropic LC phase. Ethylene glycol was used as the lyotropic solvent, enabling a wider range of CNTs types (SWNTs, DWNTs, MWNTs) to be spun than previously. Fibers spun with CNTs and nitrogen-doped CNTs were also compared [16]. The spun fibers were flexible and can be easily twisted and knotted. The orientation increased with decreasing the diameter of fibers, leading to better thermal and electrical conductivity [17].

Ericson et al. [18] improved this method by dispersing SWNTs in fuming sulfuric acid (102\% sulfuric acid), which charges SWNTs by acid anions and orders them into an aligned phase. When the viscosity reached a steady state, the SWNTs dispersion was extruded through a small capillary tube into a coagulation bath and collected onto a Teflon drum after washing (Fig. 2c-e). The resulting CFs possess good mechanical (120 GPa for $E$ and $116 \mathrm{MPa}$ for $\sigma)$ and electrical $\left(5000 \mathrm{~S} \mathrm{~cm}^{-1}\right)$ properties.

\subsubsection{Polymer-Free Spinning Method}

Unlike the LC spinning method, polymer-free spinning process using dilute, low-viscosity CNT dispersions 

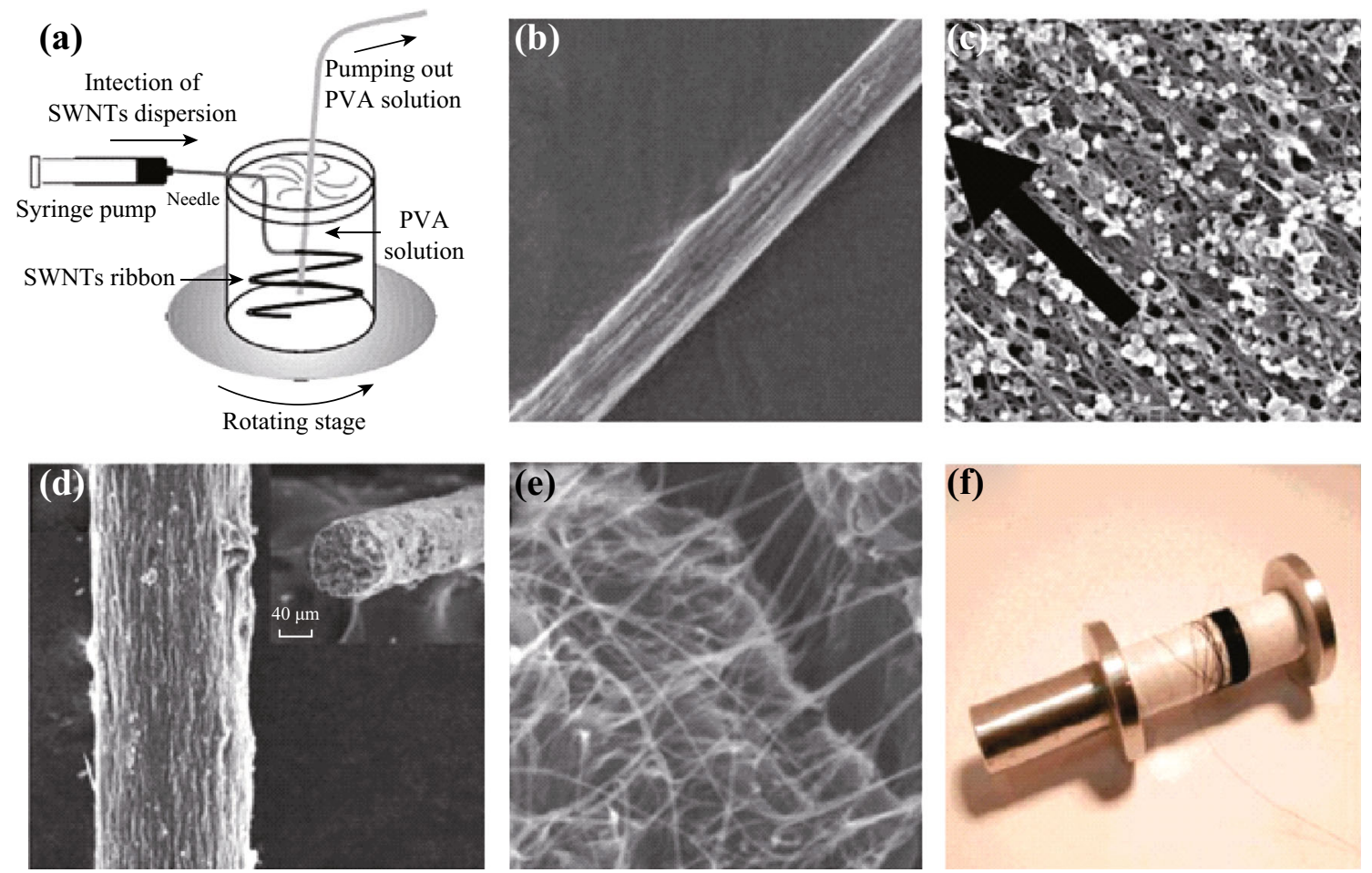

Fig. 1 a Schematic of the experimental setup for spinning CFs using polymer-based coagulation spinning method. SEM images of $\mathbf{b}$ a CF and c a ribbon (black arrow indicates the main axis) deposited on a substrate. SEM images of DNA-SWNT fibers $\mathbf{d}$ (The insert image show the round cross section) and e a magnified image of the cross section of an SWNT bundle coated with DNA. f A 10-m-long MWNT fiber collected on a small winder. a-c Reproduced with permission from Ref. [6]. Copyright 2015, American Association for the Advancement of Science. d, e Reproduced with permission from Ref. [8]. Copyright 2008, Wiley-VCH. f Copyright Reproduced with permission from Ref. [11]. Copyright 2005, American Chemical Society

$(0.6 \mathrm{wt} \%)$ as spinning dope. By adjusting the $\mathrm{pH}(\mathrm{pH}>13$ or $\mathrm{pH}<1$ ), polymer-free, surfactant-stabilized CNTs were flocculated/coagulated rapidly and uniformly to afford continuous CNTs gel fibers (Fig. 3a, b) [19]. In addition, hollow fibers, folded ribbon fibers, and solid CFs can also be easily achieved by this method (Fig. 3c-f) [19]. Due to the absence of polymer, the electrical conductivities $\left(15 \mathrm{~S} \mathrm{~cm}^{-1}\right.$ ) of these fibers were two orders of magnitude higher than that of fibers made by polymer-based coagulation spinning method $\left(0.2 \mathrm{~S} \mathrm{~cm}^{-1}\right)$.

\subsubsection{Dip-Coating Method}

Besides the above three spinning techniques, a quite simple dip-coating method of fabricating $\mathrm{CFs}$ without any additive was developed with no additional electrical equipment or complex apparatus. This method utilizes microfluidics, which includes capillary condensation, capillary flow, and surface tension, and results in the self-assembly and selfalignment of SWNT colloids (Fig. 4) [20]. The resulting SWNT fibers with a diameter of 10-20 $\mu \mathrm{m}$ and a length of several tens of centimeters possess good electrical conductivity of $3000 \mathrm{~S} \mathrm{~cm}^{-1}$.

\subsection{Solid-State Spinning Approaches}

\subsubsection{Spinning $1 D$ CFs from CNT Array}

Drawing CNTs out from superaligned CNT array is an efficient way to prepare long CFs. The original work was done by Fan and co-workers. They produced a continuous yarn of pure CNTs (Fig. 5a) by pulling out a bundle of CNTs from a CNTs array several hundred micrometers high and grown on a silicon substrate. Figure $5 b$ shows a $100-\mu \mathrm{m}$-high, freestanding CNT array with an indentation region that is being turned into a $30-\mathrm{cm}-$ long and $200-\mu \mathrm{m}$ wide yarn. It was estimated that an array area of roughly $1 \mathrm{~cm}^{2}$ can generate about $10 \mathrm{~m}$ of yarn. To achieve continuous yarns, the CNTs in the arrays must be superaligned and held together by van der Waals interactions to form bundles (Fig. 5c). The as-spun yarns were composed of parallel threads that have diameters in the range of several hundreds of nanometers (Fig. 5d) [21].

Inspired by this report, Zhu's group has done a series of work in the fabrication of CFs with high strength [22-26]. They synthesized a series of CNT arrays with lengths up to $4.7 \mathrm{~mm}$ by an ion-beam assisted deposition technique. The 


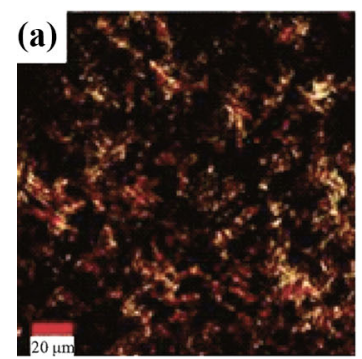

(b)

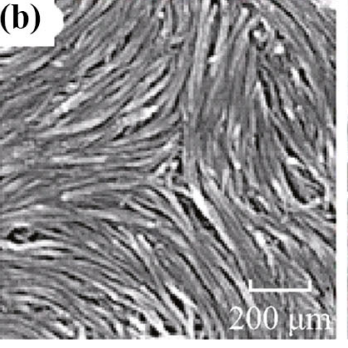

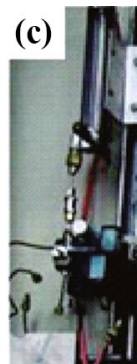
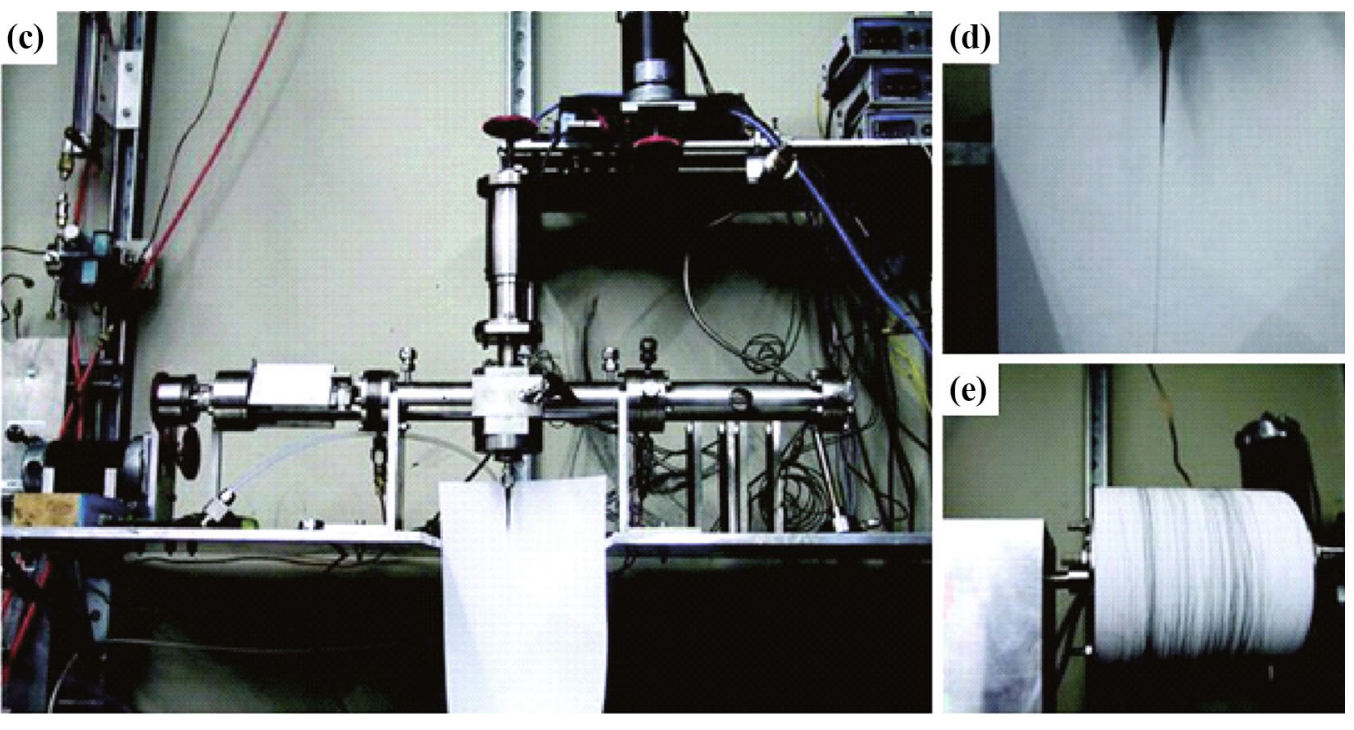

Fig. 2 a Polarizing optical microscopy (POM) observation of the SWNTs dispersed in $123 \%$ sulfuric acid. b Field emission gun scanning electron microscope (FEGSEM) image of $\mathbf{a}-1 / 2$ disclination in the nematic phase for CNTs. $\mathbf{c}$ The apparatus used for mixing and extruding neat SWNT fibers. d A jet of SWNT dispersion being extruded from a capillary tube. e A 30-m-long spool of water-coagulated SWNT fiber. a, b Reproduced with permission from Ref. [13, 14]. Copyright 2006, American Chemical Society. c-e Reproduced with permission from Ref. [18]. Copyright 2004, American Association for the Advancement of Science

CNT arrays were spinnable in a wide range of lengths $(0.5-1.5 \mathrm{~mm})$, much longer than those reported previously [22]. Such long arrays rendered CFs with superior strength and electrical conductivity, and the highest $\sigma$ of $3.3 \mathrm{GPa}$ for CFs were spun from the 1-mm array [22]. The properties of CFs can be also improved by decreasing the diameter of the fibers or increasing the packing density of CNTs in the fiber (Fig. 5e, f) [23, 24]. As a result, the CFs can be twisted to hold a 0.1-mm-diameter Ni wire (Fig. $5 \mathrm{~g}$, h).

In addition, the effect of chemical post-treatments was also demonstrated. The covalent bonding of gold nanoparticles to the CFs remarkably improves conductivity, whereas annealing CFs in hydrogen-containing atmosphere leads to a dramatic decrease in conductivity [25]. Besides common CNT constitution, well-aligned, pearllike CNTs were also assembled into macroscopic fibers. Due to the unique morphology, these fibers possess tensile strength of $350 \mathrm{MPa}$ and electrical conductivity of $250 \mathrm{~S} \mathrm{~cm}^{-1}$ [26].

\subsubsection{Direct Spinning Based on Chemical Vapor Deposition (CVD) Technique}

CVD technique is a common approach to synthesize CNTs. In 2002, the CVD technique was modified to directly synthesize microscopic 1D CFs. Long CNT strands $(20 \mathrm{~cm}$ in length, a diameter of $0.3 \mathrm{~mm}$, Fig. $6 \mathrm{a}, \mathrm{b}$ ) consisting of aligned SWNTs (Fig. 6c) were achieved through the catalytic pyrolysis of n-hexane with an enhanced vertical floating technique [27]. The edges of the spun SWNT strands were smooth, with a few individual nanotube bundles protruding out of the edge (Fig. 6b). High-resolution transmission electron microscopy (HRTEM) images (Fig. 6d) show that each bundle is composed of aligned SWNTs.

This method was then carried forward by Windle's group [28-31]. They drew the CVD-grown CNT aerogel onto a rotating rod, produced continuous fibers with a degree of twist (Fig. 6e-i). This endowed the CNT fibers with excellent flexibility. The fibers can be unwound from the spindle and wound up onto another rod and can hold the twisting state after removal from the rod (Fig. 6j) [28]. Because the structure of the fibers itself consists of a network of smaller filament subunits (nanotubes and bundles), they can bend through very tight radii without apparent permanent damage and showed knot efficiencies of $100 \%$ (Fig. 6k, 1) [29]. By optimizing the process conditions including using three different hydrocarbons, changing the concentration of iron nanocatalyst, the maximum $\sigma$ of CFs was reached to $1.46 \mathrm{GPa}$ [30].

Based on these prior works, $\mathrm{Li}$ et al. further advanced this approach and fabricated novel continuous CFs with a multilayered structure. The CFs, featured with a length of over several kilometers and a quality close to conventional textile yarns, can be controlled to be either hollow or monolithic with compacted or detached CNT monolayers by controlling the spinning process [32]. 

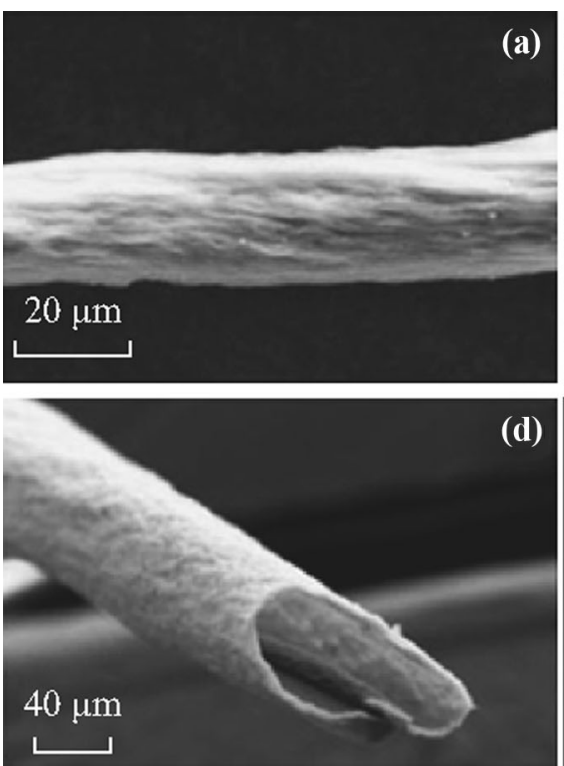
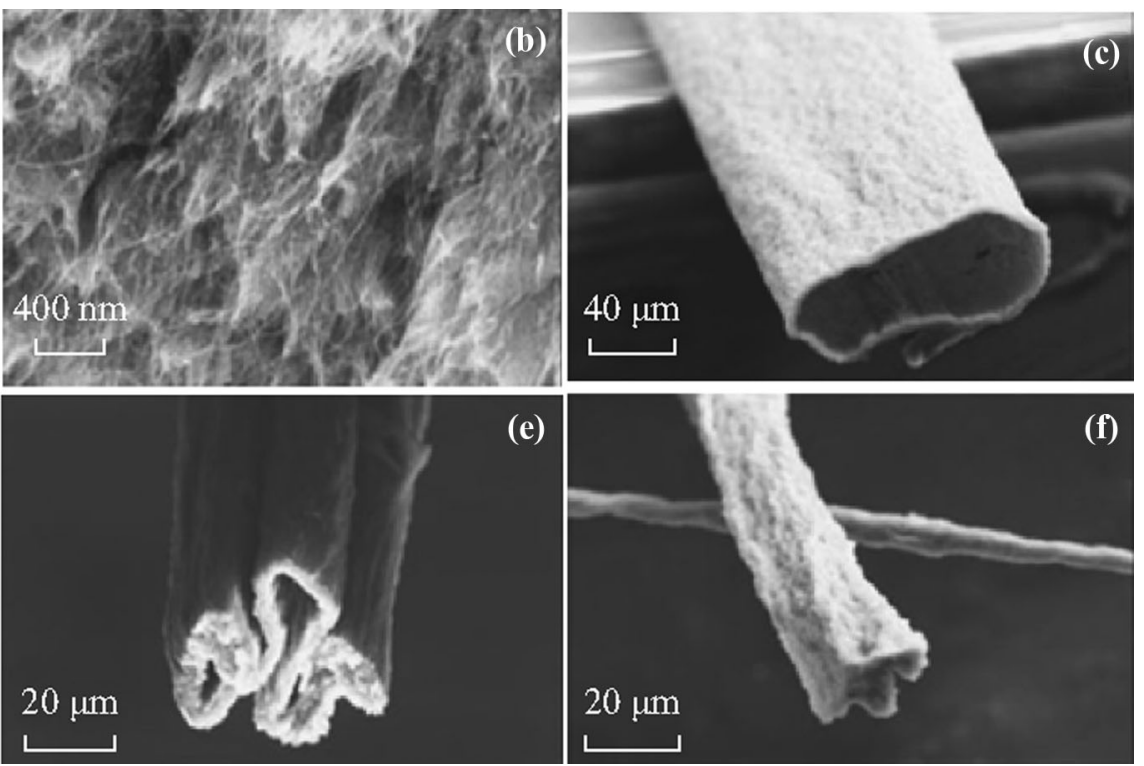

Fig. 3 SEM images of a fiber at low a and high $\mathbf{b}$ magnification. Cross sections of hollow (c, $\mathbf{d})$, folded ribbon $\mathbf{e}$ and solid $\mathbf{f}$ fibers. Reproduced with permission from Ref. [19]. Copyright 2005, Wiley-VCH

\subsubsection{Twisting CNT Films}

(a)

(b)
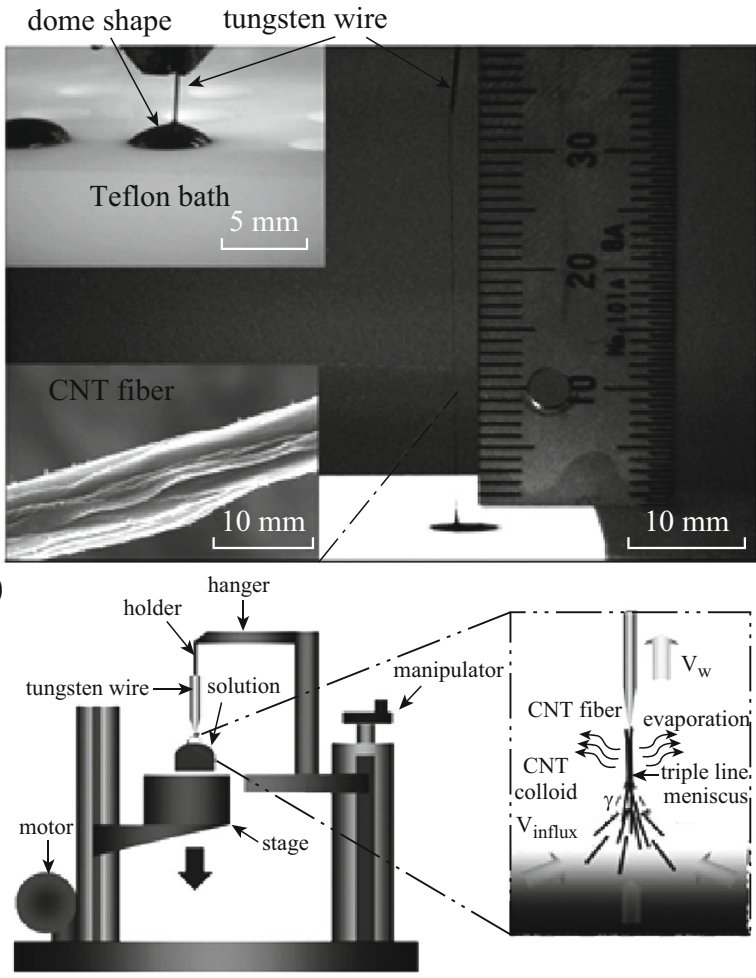

Fig. 4 a Optical photograph showing the tungsten wire immersed in a SWNT colloidal solution (upper inset; scale bar $5 \mathrm{~mm}$ ), the SWNT fiber being formed during the withdrawal process (main panel; scale bar $10 \mathrm{~mm}$ ). SEM image of the lower inset showing the magnified SWNT fiber in the dotted circle (scale bar $10 \mathrm{~mm}$ ). b Schematic of the experimental setup and the formation mechanism of the SWNT fiber. Reproduced with permission from Ref. [20]. Copyright 2009, Wiley-VCH
Twisting CNT thin films with mesh-like structure can afford uniform CF (Fig. 7a). Although this method may not be suitable for continuous production of CFs, the control of the diameters and twisting degrees is more convenient [33-35]. Since the twisting process led to the stretch and alignment of nanotubes (Fig. 7b), the interbundle junctions were reinforced and the fibers have higher strengths than the films [33]. Twisting the fibers to a higher degree, a yarn-derived spring-like CNT rope, that consisted of uniform, neat loops with perfect arrangement over long distance, were formed (Fig. 7c, d) [36]. The elongation at break point of these CNT ropes is up to $285 \%$ by loop opening and straightening during elongation. Within a moderate strain (20\%), the CNT rope behaved like a spring with a stable spring constant when stretched by 1000 times with energy absorption during contraction [36-38]. This twisting-spinning process was further employed to produce a highly twisted yarn-derived double-helix structure. The double-helix configurations were stable and consisted of two single-helical yarn segments, with controlled pitch and much larger strain up to $150 \%$ [39].

Besides the spinning of neat $\mathrm{CFs}$, this method can make the powders and nanofibers spinnable. Combined CNT sheets with other functional materials, more than $95 \mathrm{wt} \%$ of powders or nanofibers were incorporated into scrolled CNT yarns, while maintaining the guest functionality in the meantime (Fig. $7 \mathrm{~h}-\mathrm{j}$ ). The complex structures of scroll sacks were related to twist-dependent extension of Fermat spirals (Fig. 7e), Archimedean spirals (Fig. 7f), or spiral 


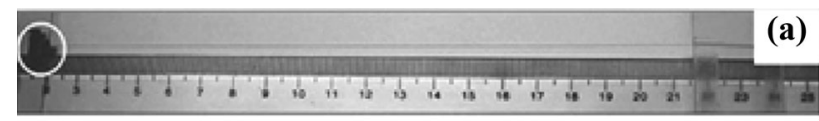

(b)

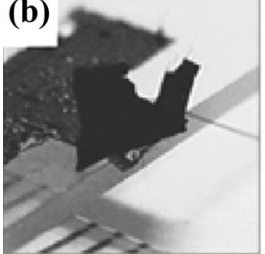

(c)
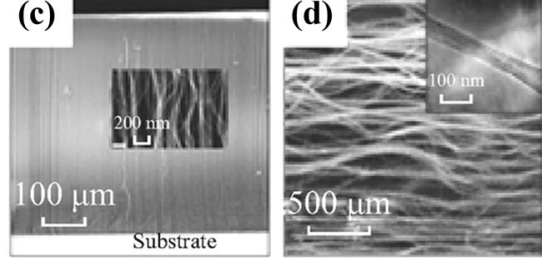

(e)

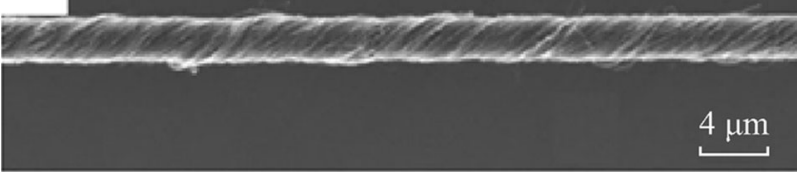

(f)
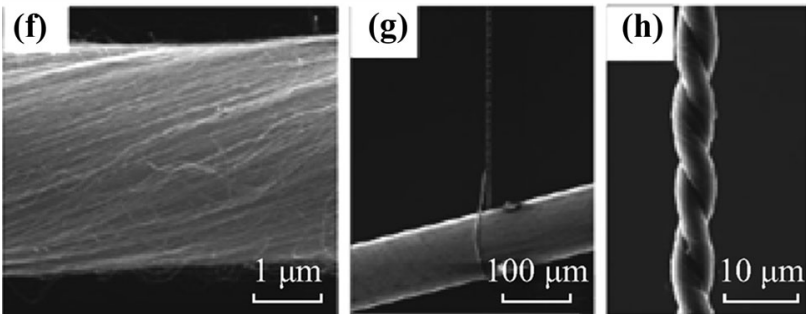

Fig. 5 a A CF pulled out from a freestanding CNT array, $\mathbf{b}$ magnified images and SEM images $\mathbf{c}$ of CNT array, $\mathbf{d}$ SEM image of the yarn of a, transmission electron microscope (TEM) image of a single thread of the CFs shown in inset, SEM images of $\mathrm{CF}$ at low $\mathbf{e}$ and high f magnification, $\mathbf{g}$ CF forming a loop around a Ni wire and then twisted, h SEM image of a two-ply twisted fiber. a-d Reproduced with permission from Ref. [21]. Copyright 2002, Nature Publishing Group. e-h Reproduced with permission from Ref. [23]. Copyright 2007, Wiley-VCH

pairs (Fig. 7g) into scrolls. Although a very small amount of CNT host is employed to mechanically confine the unbonded powder guest, biscrolled yarns can still be knotted and sewn (Fig. 7k-m). This technology was used to make yarns of superconductors, lithium-ion battery materials, graphene ribbons, catalytic nanofibers for fuel cells, and titanium dioxide for photocatalysis [40].

\subsubsection{Other Methods}

Different from the method of direct spinning based on CVD technique, which involves the directly drawing and twisting of the fresh generated CNT hot aerogel, Ci et al. fabricated continuous CFs from the cool DWNT cotton based on surface-tension-driven densification. The starting, cotton-like material must be wet in order to draw a continuous fiber because the wet material is denser and has stronger internanotube interactions [41]. Figure 8a shows a schematic illustration of the spinning process, and an optical image of the as-spun DWNT fibers. Similarly, a 35-mm-long quartz-supported CNT cotton was spun into 10 -cm-long fibers with good mechanical strength through simply pulling and rotating [42]. Individual CNTs in the cotton were easily collected and aligned along the pulling direction during fiber spinning (Fig. 8b, c).

Electrophoretic approach is generally used in the field of assembly. The early try of assembling CNTs into fibers was finished by Alldredge and co-workers. A carbon fiber tip was withdrawn slowly from a dispersion of SWNT under an electric field. During this process, CNTs were gathered around and assembled one-by-one at the positively charged carbon fiber tip, yet only 5-cm-long fibers could be achieved [43]. Distinct from the former assembly method, Liu et al. invented a step-by-step way to prepare CNT yarns by drawing the CVD-grown films through a series of diamond wire drawing dies (Fig. 8d). Passing through the series dies with decreased pore diameters, the CNT networks were highly aligned and densified into 1D fibers $[44,45]$.

No matter what methods applied, the nanotubes in $\mathrm{CF}$ must be superaligned and densely packed to achieve high performance. Based on the above, we can draw a conclusion that shear rate within the extrusion orifice, flow-induced alignment in the direction of the fluid velocity and the spontaneous ordering in the liquid crystalline phase are all responsible for the alignment. Polarized Raman spectroscopy and XRD can been used to quantitatively probe the degree of alignment. Meanwhile, high-speed drawing, twisting, and surface-tension-driven densification can increase in interbundle contact and packing density.

We will give an example to show how to improve the degree of alignment and packing density by adjusting process parameters. One typical work by Windle's group systematically explored the effects of density and orientation on the properties of CNT fibers [31]. The degree of alignment and density can be increased simultaneously by increasing the fiber-winding rate, which is a basic precept for the processing of commercial polymer fiber. Additionally, running the fiber through a vapor stream can also significantly densify the fiber due to the surface tension effect. Another important processing technique to improve the mechanical performance is decreasing the diameters of the fiber, which will effectively reduce the flaws along the fiber samples according to Griffith size-scaling law.

\section{Graphene Fibers: 1D Graphene Macroscopic Architectures}

Inspired by the assembly strategies for CFs, 1D GFs were also assembled through analogous approaches such as LC spinning, twisting their 2D films, electrophoretic method. Even so, many novel methods were further developed to achieve GFs with high strength and high electrical conductivity. 

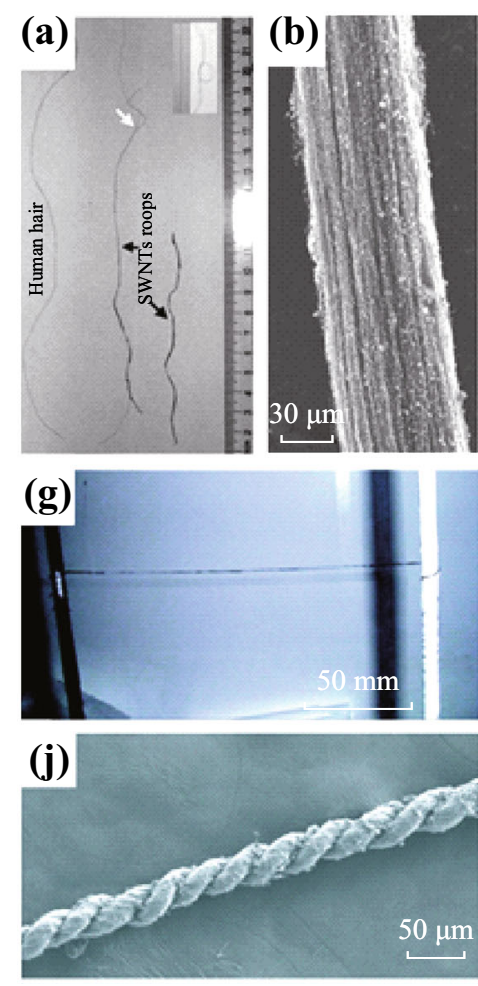
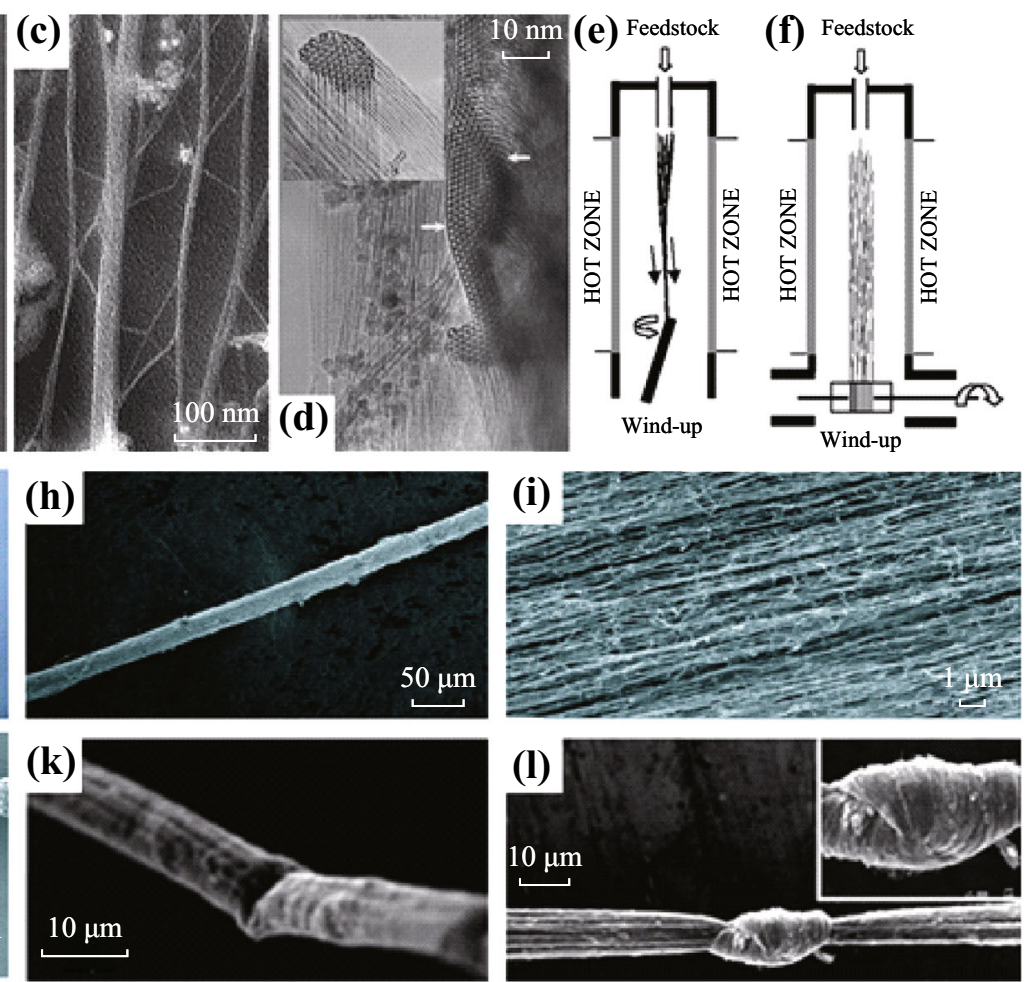

Fig. 6 a Optical image showing a human hair and two as-grown SWNT fibers. SEM images of CF at low b and high $\mathbf{c}$ magnification. d HRTEM image of a top view of a SWNT fiber. Schematic of the direct spinning process with the spindle aligned at $25^{\circ}$ (e) and normal to $\mathbf{f}$ the furnace axis. g Photograph of CFs wound from the spindle (left) onto a second spindle (right). SEM images of CF at low (h) and high $\mathbf{i}$ magnification. $\mathbf{j}$ A twisted CF after its removal from the furnace. SEM image of CFs showing a kink induced by bending (k) and showing an overhand knot (l). ad Reproduced with permission from Ref. [27]. Copyright 2002, American Association for the Advancement of Science. e-j Reproduced with permission from Ref. [28]. Copyright 2004, American Association for the Advancement of Science. k, l Reproduced with permission from Ref. [29]. Copyright 2010, Wiley-VCH

\subsection{Liquid-State Spinning Approaches}

Graphene or graphene oxide (GO) can yield LC phase due to its high aspect ratio (or width/thickness ratio) and sufficient dispersibility/solubility with the solution concentration up to $30 \mathrm{mg} \mathrm{mL}^{-1}$ [46-49]. This LC phase is promising for processing of neat graphitic macroscopic articles such as fibers. In 2011, Gao's group firstly spun continuous GF utilizing the liquid crystal phase behavior of GO sheets in aqueous solution (Fig. 9a) [46]. The gel-state graphene was extruded from glass syringes and injected into the $\mathrm{NaOH} /$ methanol solution under $1.5 \mathrm{MPa}$ held by $\mathrm{N}_{2}$ flow. The sheet alignment inherited from the intrinsic lamellar order of LCs provides strong interactions between contacted sheets that were responsible for the strong mechanical strength of GFs. The locally crumbled structures of individual sheets made the GFs flexible (Fig. 9b, c), which can be even woven into a compact knot (Fig. 9d) [46]. The GFs were then functionalized by $\mathrm{MnO}_{2}$ and polyaniline and used as electrodes of yarn supercapacitors $[50,51]$.
Wallace's group used a multi-hole spinneret to produced GO yarns (Fig. 9e, f). They revealed the correlation of processability with LC behavior (Fig. 9g), aspect ratio, and the dispersion concentration to provide a viable platform for spinning of LC GO [49]. By adjusting the GO sheet size and polydispersity, the very low concentrations GO dispersions (as low as $0.075 \mathrm{wt} \%$ ) can be used as spinning dope to prepare the continuous GFs [49]. The morphology of the fiber reveals that GO sheets were stacked in layers with some degree of folding and were orientated along the fiber axis direction (Fig. 9h, i). Neat and macroscopic GFs with mechanical strength of $182 \mathrm{MPa}$ and electrical conductivity of $3500 \mathrm{~S} \mathrm{~m}^{-1}$ were also be spun by Yu's group. They injected GO dispersion into CATB solution, and the curliness-fold formation mechanism of GO fibers was proved by SEM observations (Fig. 10) [52]. By introducing shear stress during wet-spinning process, flat and uniform ribbons with high flexibility were also produced [53].

Neat GO aerogel fibers with unique "porous core-dense shell" structure from flowing GO LCs with lamellar ordering were fabricated by the combination of spinning 

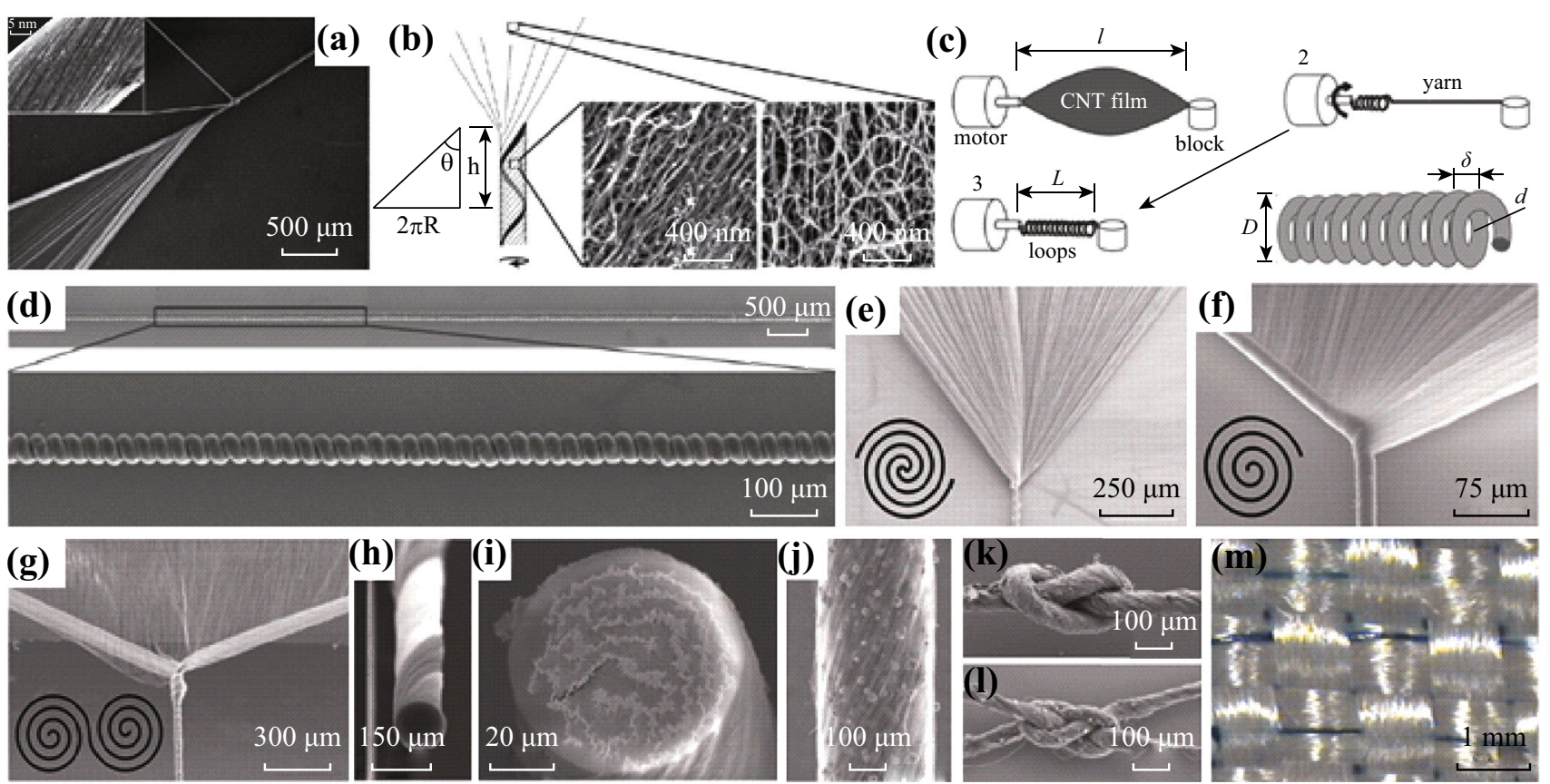

Fig. 7 a SEM image of a 200-nm-thick CF made from a CNT film with a width of $2 \mathrm{~mm}$. b The schematic image left illustrates the twisting process induced elongation for the film and the two SEM images right come from the unstrained and strained part of the film, respectively. c Illustration of the spinning process of spring-like CNT ropes. d SEM images of a 4.4-mm-long section of rope consisting of highly uniform, perfectly arranged loops. SEM images of Fermat $\mathbf{e}$, Archimedean $\mathbf{f}$ and dual Archimedean $\mathbf{g}$ type scrolls, which are illustrated in the insets, respectively. h, i SEM images of $\mathrm{Si}_{3} \mathrm{~N}_{4} \mathrm{NT} @ \mathrm{MWNT}$ biscrolled yarn. The brighter areas correspond to MWNTs. j SEM image of TiO $@$ MWNT yarn. SEM images of overhand knot in 95\% $\mathrm{LiFePO}_{4} @ \mathrm{MWNT}$ yarn $\mathbf{k}$ and carrick bend knot between two 88\% $\mathrm{SiO}_{2} @ \mathrm{MWNT}$ yarns (l). m Photograph of $85 \% \mathrm{TiO}_{2} @$ MWNT yarn sewn into Kevlar textile. a, b Reproduced with permission from Ref. [33]. Copyright 2009, WileyVCH. c, d Reproduced with permission from Ref. [36]. Copyright 2012, Wiley-VCH. e-m Reproduced with permission from Ref. [40]. Copyright 2011, American Association for the Advancement of Science

technology and ice-templating strategy (Fig. 11a) [54]. Graphene porous fibers (GPFs) were achieved after chemical or thermal reduction, which were flexible and can be folded (Fig. 11b). The folded GPFs was stretched to its original straight shape without any fracture (Fig. 11c). The SEM observation revealed that graphene sheets interconnect with each other and form axially aligned empty cells (Fig. 11d, e). The GPFs held high $\sigma$ and high compression strength [54].

Neat, morphology-defined, graphene-based hollow fibers were fabricated via a coaxial two-capillary spinning strategy [55]. The coaxial two-capillary spinneret was fabricated by inserting a stainless steel needle (connected to a syringe-containing coagulation bath) into a branched glass tube (filled with GO suspension) with a capillary tip. Figure 11f shows a schematic illustration of the apparatus for spinning with the coagulation bath of methanol solution containing $3 \mathrm{M} \mathrm{KCl}$. The semitransparent graphene oxide hollow fibers (GO-HFs) (Fig. 11g) were continuously produced, and the hollow structure was reflected by the open tip (Fig. 11g, inset) and SEM observation (Fig. 11h). After being naturally dried, the GO-HFs were converted to black color (Fig. 11i). Replacing the inner fluid of $\mathrm{KCl} / \mathrm{methanol}$ solution with compressed air, the obtained GO-HFs displayed a necklace-like structure (Fig. 11j).

This coaxial wet-spinning technology is further extended to prepare core-sheath GFs [56]. The spinning process is shown in Fig. 12a. GO dispersion and sodium carboxymethyl cellulose (CMC) aqueous solutions were chosen as inner and outer spinning dopes, respectively. The as-prepared coaxial fibers were featured with $\mathrm{GO}$ as core and CMC as sheath (Fig. 12b, c), and the CMC sheath wrapped the core tightly without any gaps and void (Fig. $12 \mathrm{~h}-\mathrm{j}$ ). Treated by chemically reduced with hydroiodic acid, the color of the core was changed from brown to dark while the polyelectrolyte sheath retained well (Fig. 12d). A 100-m-long coaxial fiber was achieved by this approach (Fig. 12e). The integration of high flexibility and scalable fabrication made these coaxial fibers promising in co-woven with cotton yarns (Fig. 12f). A cloth supercapacitor was then fabricated by using two individual 40-cm-long coaxial fibers as anode and cathode (Fig. 12g). Besides the cloth supercapacitor, the yarn supercapacitors were also prepared by tightly intertwining two coaxial fibers followed by coating a layer of $\mathrm{H}_{3} \mathrm{PO}_{4}$-PVA gel electrolyte as ion source (Fig. 12k-m). The as-prepared yarn supercapacitors were very safe, and short circuit never 


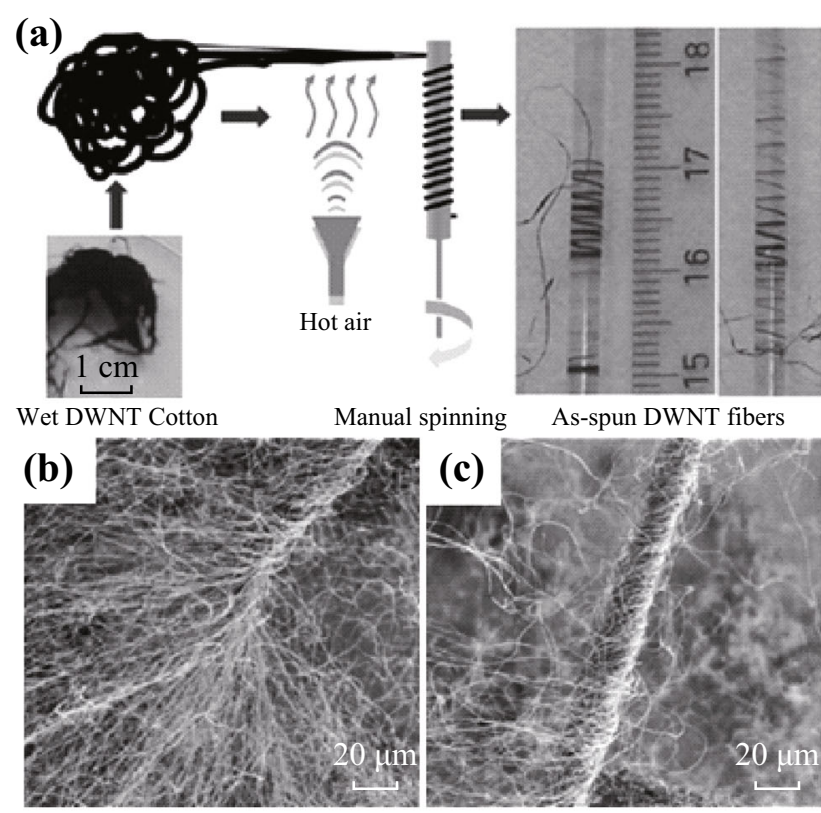

(d) $\longleftarrow$ Direction of drawing

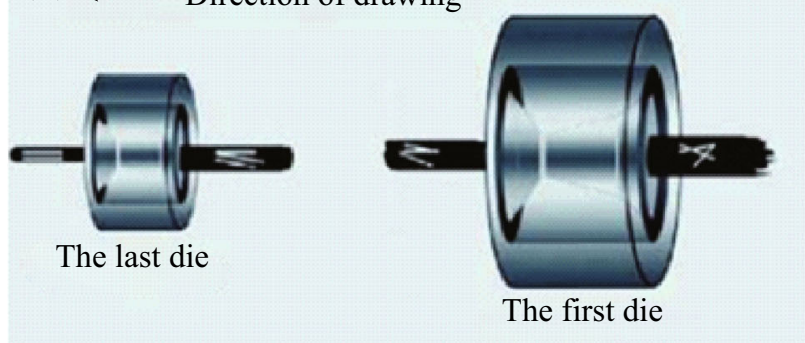

Fig. 8 a Schematic illustration of the drawing-drying spinning process and optical images of the as-spun DWNT fibers. b, c SEM images of CNT cotton based fibers. d Schematic of fiber shaping by drawing CNT films through a series of diamond wire drawing dies. a Reproduced with permission from Ref. [41]. Copyright 2007, Wiley-VCH. b, c Reproduced with permission from Ref. [42]. Copyright 2007, Wiley-VCH. d Reproduced with permission from Ref. [44]. Copyright 2008, American Chemical Society

happened in the assembly and working process of YSCs because of the protection of the CMC sheath.

Chemical functionalization of graphene with polymer can enhance the dispersibility in specific solvents and afford them LC behavior. Polyacrylonitrile (PAN) and poly (glycidyl methacrylate) (PGMA) chains were covalently and uniformly grafted onto GO surfaces via a simple free radical polymerization process $[57,58]$. The continuous, conductive, flexible graphene composite fibers were then spun utilizing them as building blocks.

To make the multifunctionalization of graphene-based fibers fast and easy, Gao's group combined liquid crystal self-templating (LCST) approach and wet-spinning technology to continuously fabricate biomimetic composites fibers (Fig. 13a-g). They introduced hyperbranched molecules (HPG) [59, 60] into the LC-state GO dispersion to achieve nacre-mimic fibers with excellent mechanical performance ( $\sigma=652 \mathrm{MPa}, E=20.9 \mathrm{GPa}$ ). A deformation mechanism model was also proposed based on the SEM observation (Fig. 13h-j). Besides HPG, PVA [61] and sodium alginate (SA) [62] acted as guests were also mixed with GO host. The assembled macroscopic GO-SA composite fiber inherited the alignment of the GO sheets from the LC phase (Fig. 13k-m), reached $784.9 \mathrm{MPa}$ and $58 \mathrm{GPa}$ for $\sigma$ and $E$, respectively. Apart from the above macromolecules, inorganic components such as Ag nanowires [63], bismuth oxide nanotubes [64] and montmorillonite [65] were also employed as introduced guests to endow graphene-based composite fibers with multiple functions such as high electrical conductivity, improved electrochemical properties and flame retardant.

\subsection{One-step Dimensionally Confined Hydrothermal Strategy}

Besides the above wet-spinning method, Qu's group developed a one-step dimensionally confined hydrothermal strategy to prepared GFs [66]. They injected aqueous GO suspension into the glass pipeline with a $0.4-\mathrm{mm}$-inner diameter by use of a syringe and baked it at $230{ }^{\circ} \mathrm{C}$ for $2 \mathrm{~h}$ after sealing up the two ends of the pipeline. The wet state GF matching the pipe geometry was produced. It was released from the pipeline by flow of $\mathrm{N}_{2}$ and was hold by glass slides for drying in air [66]. To endow the fiber multifunction, they integrated the GFs with $\mathrm{Fe}_{3} \mathrm{O}_{4}$ and $\mathrm{TiO}_{2}$ and obtained functional GFs with magnetic and photoelectric (electrical current induced by light) response [66]. The $\mathrm{Fe}_{3} \mathrm{O}_{4}$ functionalized GFs was then acted as both substrate and catalyst for CVD growth of CNTs. The subsequent 1D CNTs and 2D graphene hybrid fibers with a large surface area (ca. $79.5 \mathrm{~m}^{2} \mathrm{~g}^{-1}$ ) and high electrical conductivity $\left(1200 \mathrm{~S} \mathrm{~m}^{-1}\right)$ were achieved [67]. This strategy is then extended to using PP pipeline instead of glass pipeline and vitamin $\mathrm{C}$, and low temperature chemical reduction replace of hydrothermal reduction [68].

\subsection{Electrophoretic Self-Assembly Method}

Reduced GO nanoribbon (GONR) fibers were also fabricated by using an electrophoretic self-assembly method without any polymer or surfactant. Specifically, a graphitic tip as a positive electrode was immersed into the chemically reduced GONR colloidal solution in a Teflon vessel containing the counter electrode. The distance between two electrodes is ca. $5 \mathrm{~mm}$, and the fiber is drawn out under a constant voltage ranging from 1 to $2 \mathrm{~V}$. After annealing treatment, the fiber showed superior field emission performance with a low potential for field emission and a giant field emission current density when compared with vertically aligned few-layer graphene and 

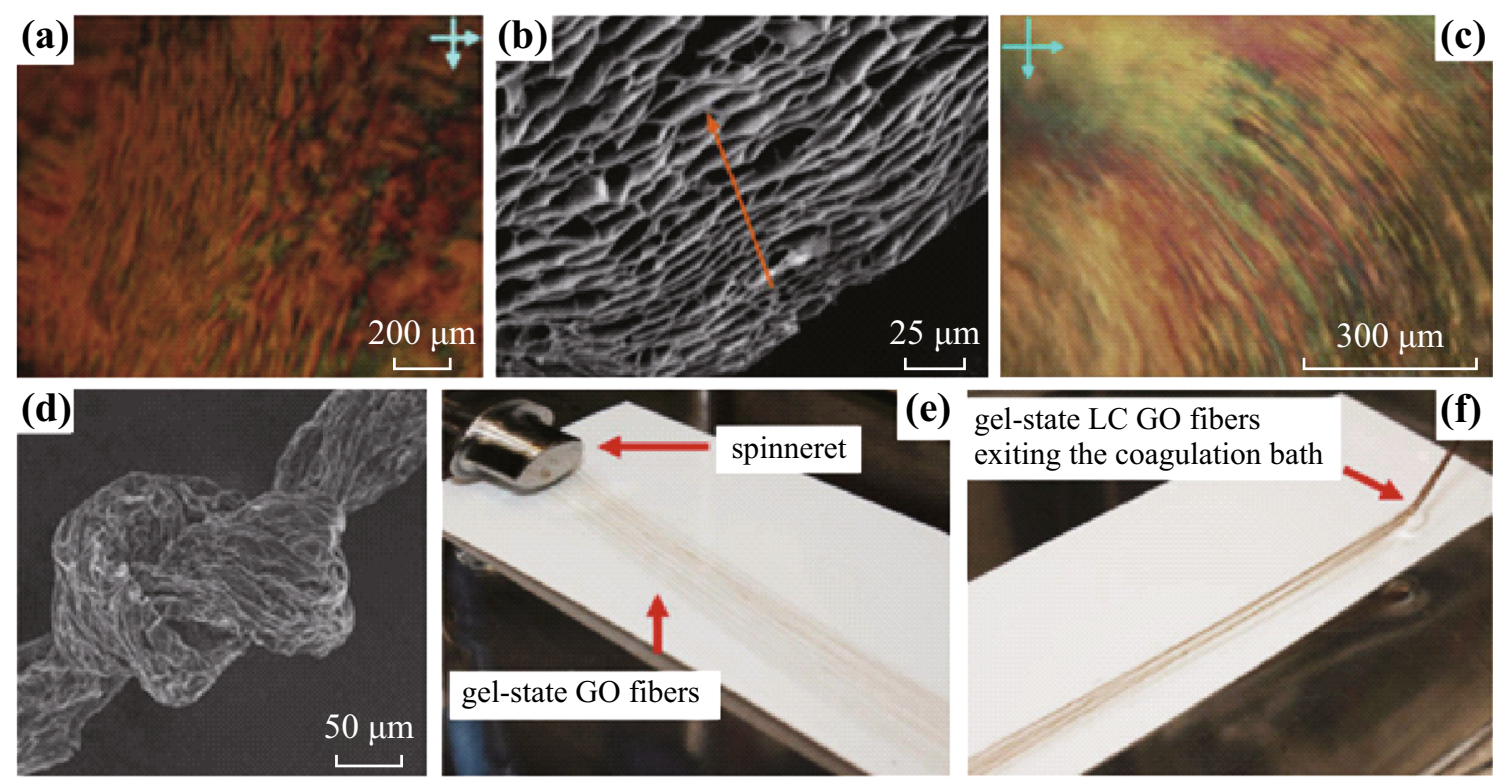

(e)
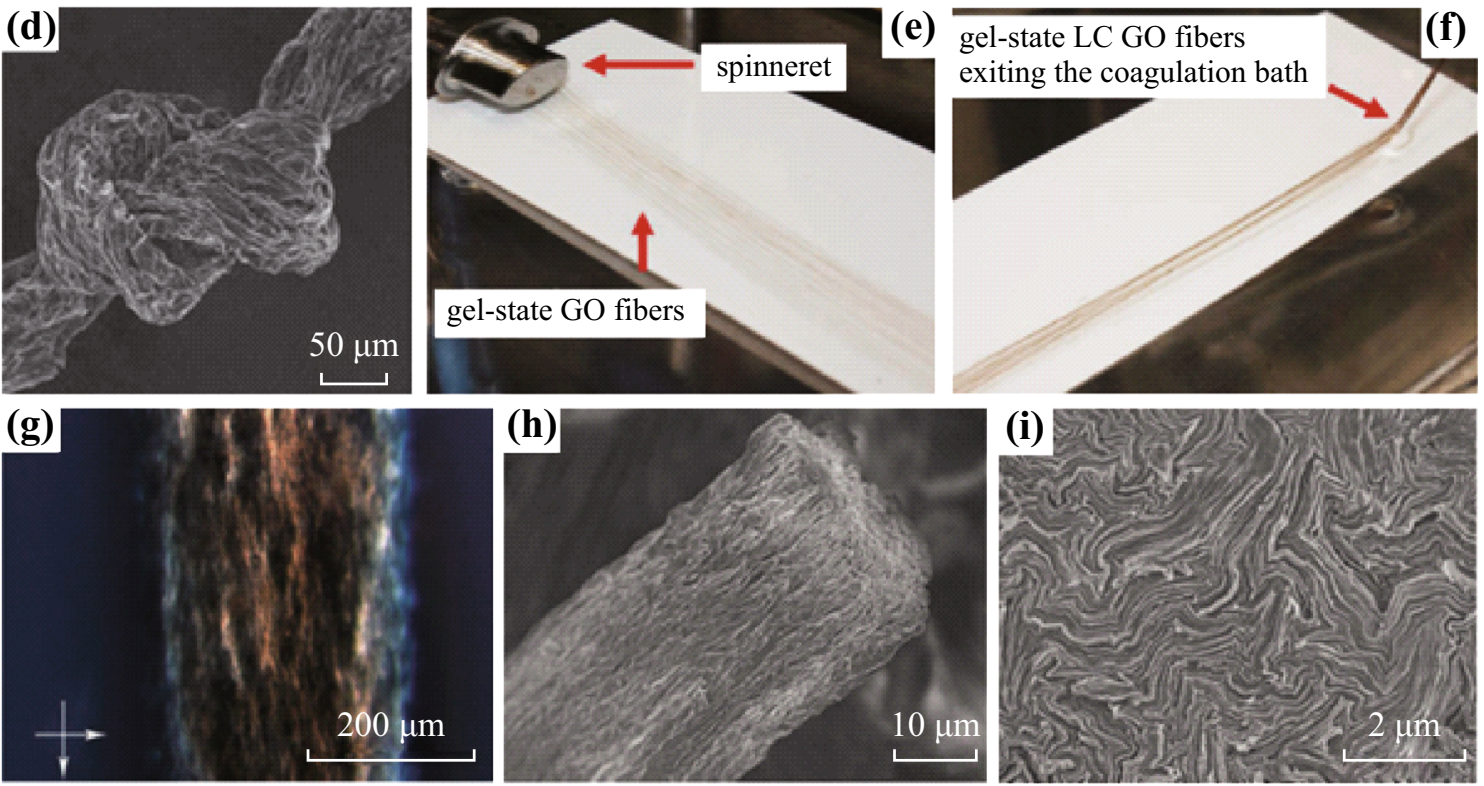

Fig. 9 a POM observation of GO aqueous dispersions with a fingerprint texture of chiral phase. b, c Cryo-SEM images and POM textures for GO chiral LCs. $\mathbf{d}$ a tight knot of GF. e, $\mathbf{f}$ photographs of GO yarns produced using a multi-hole spinneret. $\mathbf{g}$ POM observation of gel-state GO fiber showing birefringence. SEM images of a GO fiber $\mathbf{h}$ and its magnified cross section (i). a-d Reproduced with permission from Ref. [46]. Copyright 2011, Nature Publishing Group. e-i Reproduced with permission from Ref. [49]. Copyright 2013, Wiley-VCH

(A) (a)

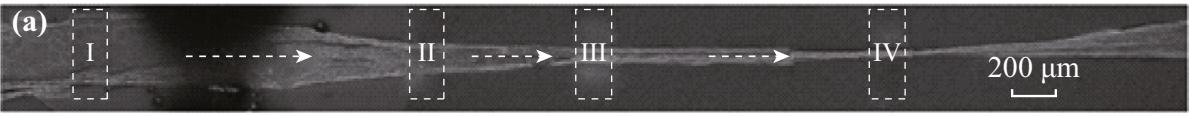

(b)
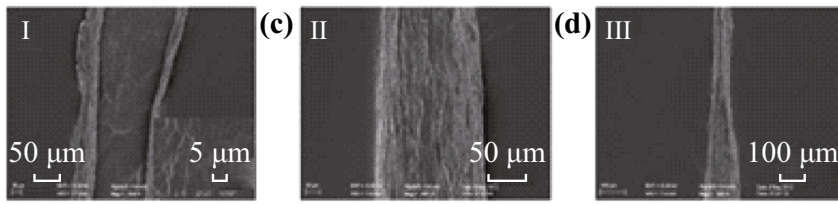

(B)

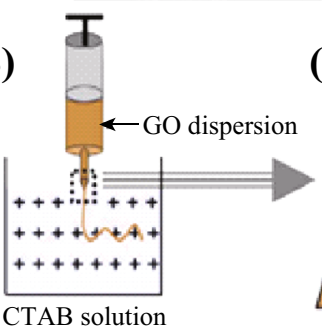

(C) GO film

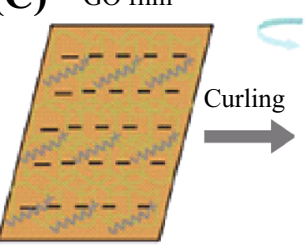

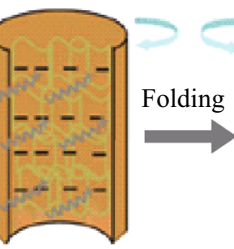

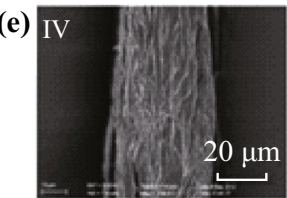

GO fiber
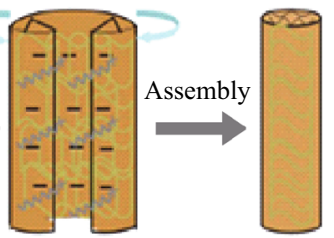

Fig. 10 A SEM images illustrating the assembly process of the GO fiber. Schematic illustration of the apparatus $\mathbf{B}$ and assembly mechanism C of wet-spinning GO fibers. Reproduced with permission from Ref. [52]. Copyright 2012, Nature Publishing Group 

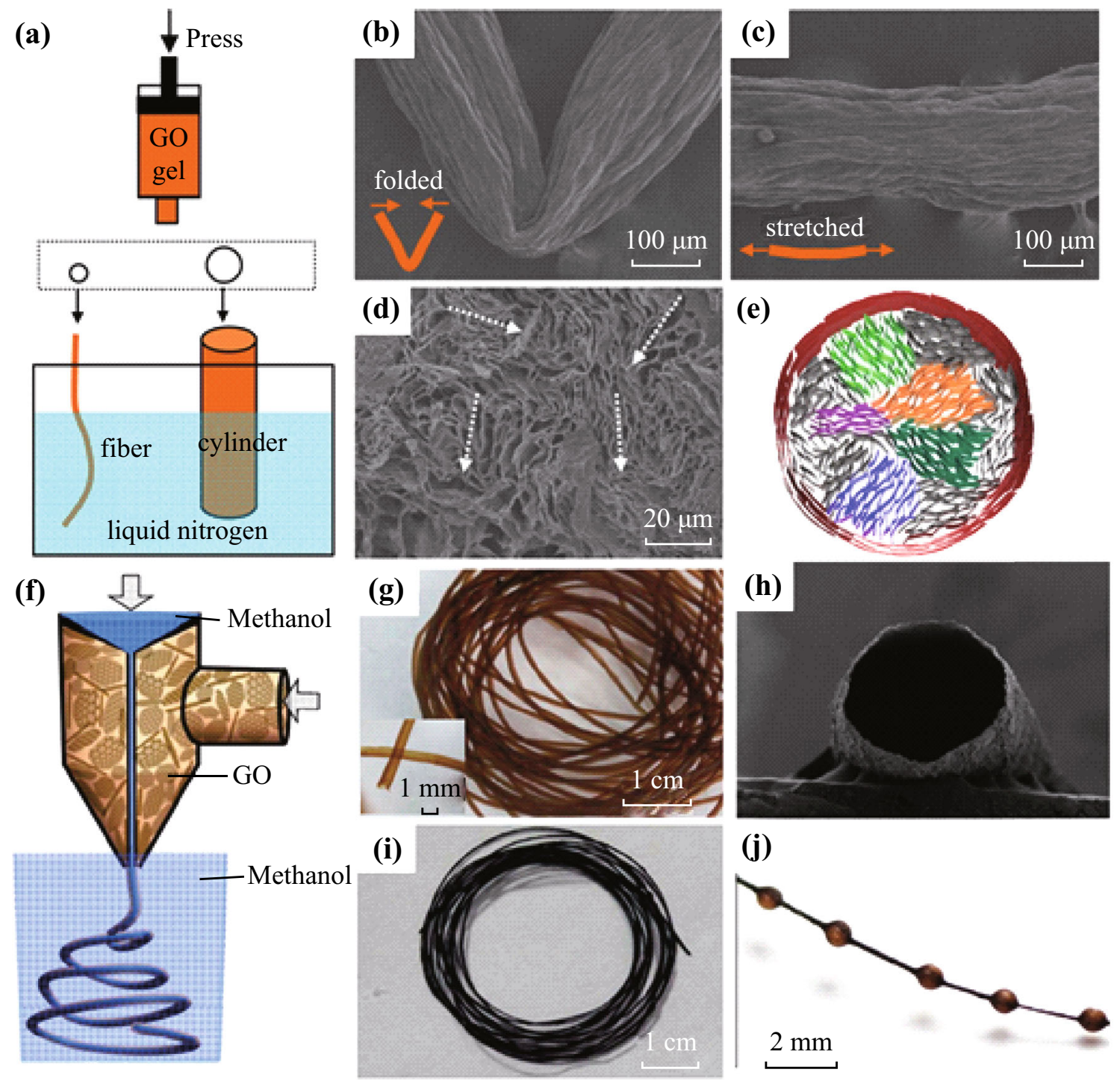

Fig. 11 Scheme for preparation of GO porous fibers a and hollow fibers (f). SEM images of folded $\mathbf{b}$, stretched $\mathbf{c}$ and fracture morphology $\mathbf{d}$ of GPFs. e Schematic core-shell structure model for GPFs. Photographs of GO-HFs in coagulation bath g and the naturally dried GO-HFs (i). h SEM images of naturally dried GO-HF. j A Photograph of necklace-like GO-HF. a-e Reproduced with permission from Ref. [54]. Copyright 2012, American Chemical Society (f-j). Reproduced with permission from Ref. [55]. Copyright 2013, American Chemical Society

single-layer graphene films prepared by electrophoretic deposition [69].

\subsection{Self-Assembly from 2D Graphene Films}

Different from the above three methods using GO as precursors, self-assembly method directly utilizes graphene films to fabricate GFs. The 1D fiber-like structures were selfassembled from 2D graphene films in an organic solvent (e.g., ethanol, acetone) and then dried to give the porous and crumpled structure [70, 71]. The morphology and pore structure of the fibers can be adjusted by controlling the evaporation of solvents with suitable surface tension [72-74].

\section{Mechanical Properties of CNTs/Graphene Fibers}

\subsection{Mechanical Properties of CFs}

Based on the assembly approaches, the mechanical properties of CFs show a broad range from 109 to $8800 \mathrm{MPa}$ for $\sigma$ and 1.25 to $397 \mathrm{GPa}$ for $E$ (as shown in Table 1). In general, CFs spun from CNT aerogel/array show higher $\sigma$ and $E$ than those produced by wet-spinning approach. To further improve the mechanical performance to compete with conventional high-performance fiber materials (e.g., Kevlar and CFs), it is fundamentally important to probe the critical factors affecting the mechanical properties of CFs. 

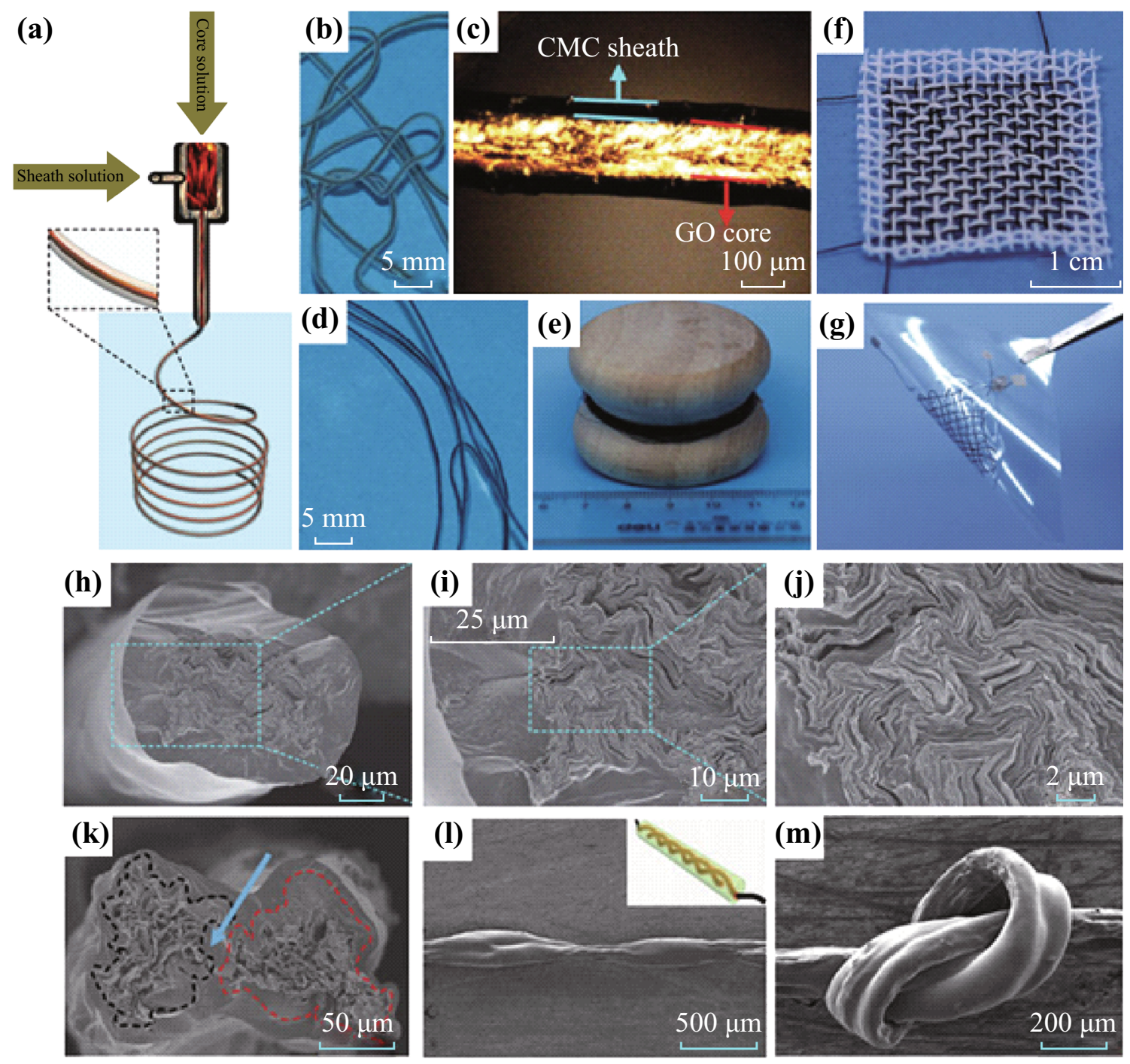

Fig. 12 a Schematic illustration showing the coaxial spinning process. The magnified image of wet GO@CMC fiber b and GO@CMC fiber (d). c POM image of wet GO@CMC fiber indicating the core-sheath structure and the well-aligned GO sheets in the core part. e The macroscopic photograph of the RGO@CMC coaxial fiber. f Two intact coaxial fibers woven with cotton fiber. $\mathbf{g}$ Supercapacitor device based on the cloth fabricated by two coaxial fibers. $\mathbf{h}-\mathbf{j}$ SEM images of coaxial fibers. SEM images of cross-sectional $\mathbf{k}$ and side $\mathbf{l}$ view of a two-ply YSC. Inset of i shows the schematic illustration of YSC. m SEM image of a two-ply YSC knot. Reproduced with permission from Ref. [56]. Copyright 2014, Nature Publishing Group

The CNT structures (diameter, aspect ratio, defects, morphology, purity, and orientation) [75] and CF geometries (e.g., diameter, twist angle, and volume fraction) are two major factors dominating CFs' mechanical behavior. In the case of CNT structures, SWNTs or DWNTs are the best candidates to achieve good fiber performances compared with MWNTs. The well-aligned CNT arrays yield fibers with much higher performance, while wavy and entangled arrays give poor properties [76]. The larger the aspect ratio of graphene units and CNTs, the higher the tensile strength of the CF. In the case of fiber geometries, both the $\sigma$ and $E$ would reduce with increasing twisting MWNT fibers to a larger extent [77].
Owing to the weak van der Waals interaction between the neighboring CNT, the CNT can easily slide on each other, resulting in a low $E$ of CFs. To solve this problem, strong interaction between the tubes must be introduced. Electron beam irradiation is an effective approach to cause stable links between neighboring CNT within bundles. The mechanical properties showed a 30 -fold increase in the bending modulus due to the formation of stable crosslinks that effectively eliminate sliding between the nanotubes [78].

Besides the electron-beam-induced crosslinking approach, a method based on filtration-twisting was proposed to fabricate CNT-based composite fibers [35]. The 
(a)

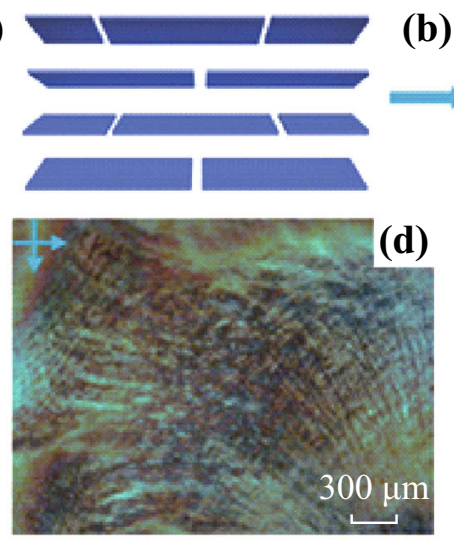

(h)
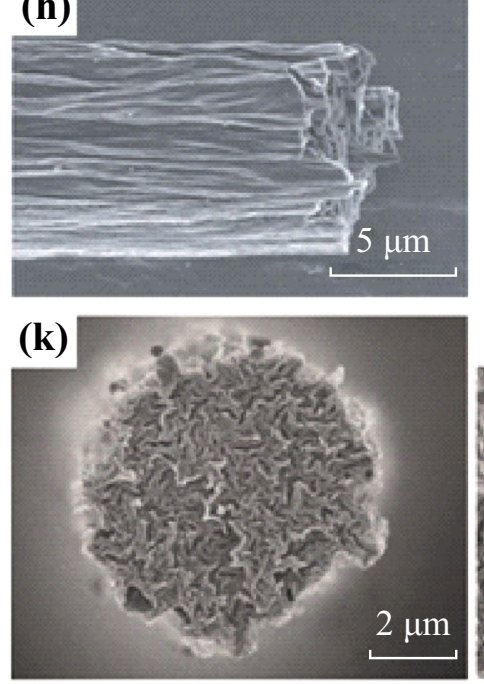
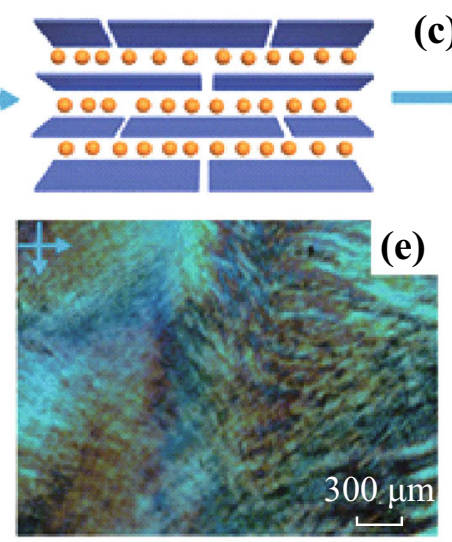

(c)
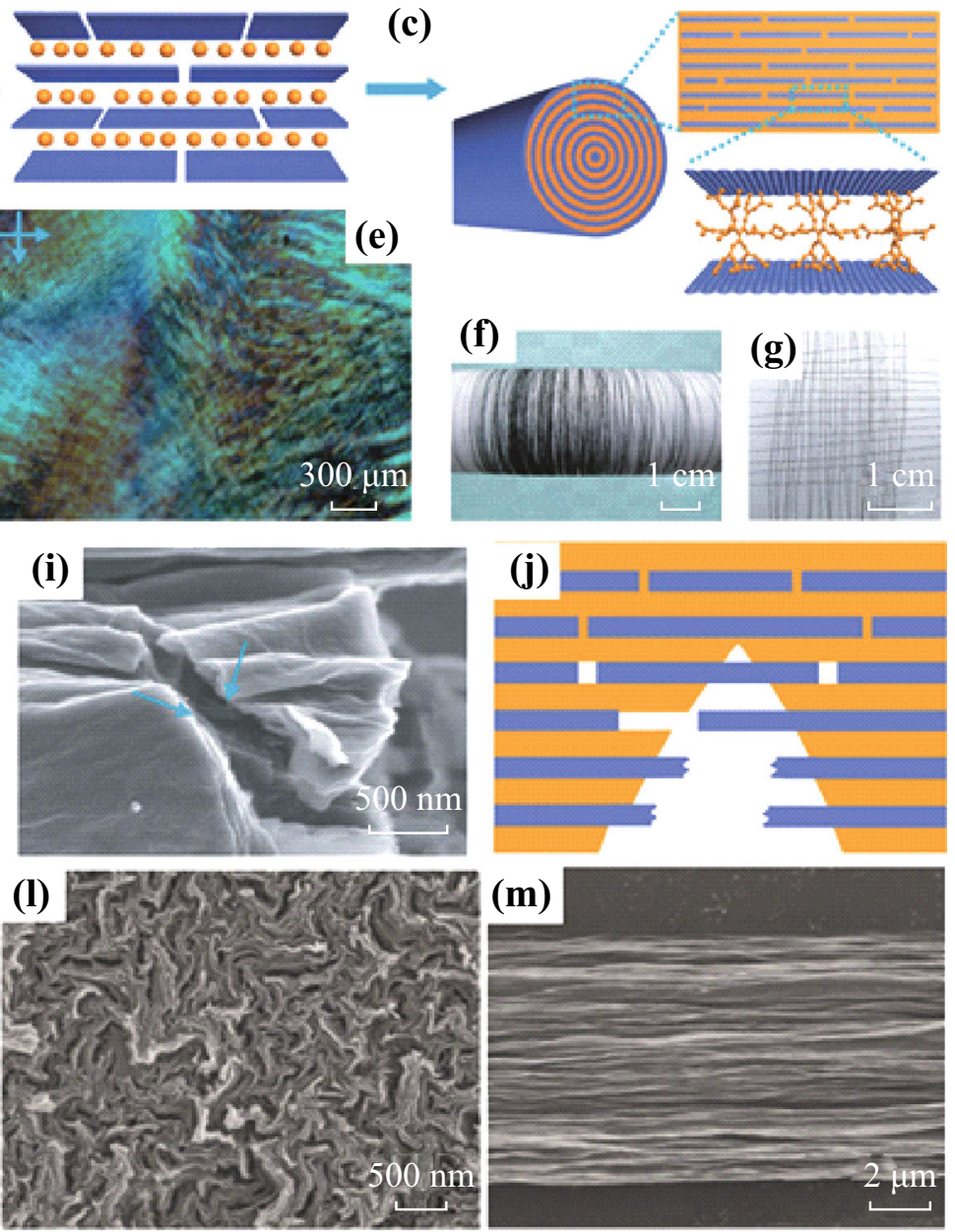

Fig. 13 Schematic illustration of LCST strategy a-e and the resulting composite fibers (f, $\mathbf{g}$ ). Morphology of fracture section of GO-HPG fibers $\mathbf{h}, \mathbf{j}$ and deformation mechanism model under tensile. SEM images of the cross section $\mathbf{k}, \mathbf{l}$ and lateral view $\mathbf{m}$ of a GO-SA fiber. $\mathbf{a}-\mathbf{j}$ Reproduced with permission from Ref. [60]. Copyright 2014, Nature Publishing Group. k-m Reproduced with permission from Ref. [62]. Copyright 2016, Springer

strength values for epoxy- and PVA-infiltrated fibers were increased to $0.9-1.6$ and $0.7-1.3 \mathrm{GPa}$, while modules were improved to $30-50$ and $20-35 \mathrm{GPa}$, respectively. The toughness of PVA-infiltrated fibers reaches $50 \mathrm{~J} \mathrm{~g}^{-1}$, far superior to commercially used high-strength fibers [35]. Further increased toughness results from combining CNTs and reduced GO flakes in solution-spun polymer fibers. The gravimetric toughness approaches $1000 \mathrm{~J} \mathrm{~g}^{-1}$, far exceeding spider dragline silk $\left(165 \mathrm{~J} \mathrm{~g}^{-1}\right)$ and Kevlar (78 $\mathrm{J} \mathrm{g}^{-1}$ ) [79].

Apart from PVA or epoxy, super-strong CFs with tensile strength up to GPa scale were also fabricated inspired by the molecular mechanics of mussel adhesive formation (Fig. 14a, b). The CFs were spun from the CNT forest (Fig. 14c, d). Treated with twisting, infiltrating the adhesive polymer poly(ethylenimine) catechol (PEI-C), and solvent evaporation, the strong and densified fibers (Fig. 14e, f) were prepared. The PEI-C-infiltrated CFs
(PEI-C-CNT) exhibited 65\% increase in $\sigma$ from 0.55 to $0.91 \mathrm{GPa}$ compared with the pure fibers. After crosslinking at $120{ }^{\circ} \mathrm{C}$ for $2 \mathrm{~h}, \sigma$ and $E$ of the fibers increased to 2.2 GP and $120 \mathrm{GPa}$, respectively (Fig. 14g). Besides the heatinduced crosslinking, the Fe-catechol coordination and Feinduced oxidative crosslinking chemistry further enhanced $\sigma$ to $2.5 \mathrm{GPa}$ and toughness of $32.5 \mathrm{MJ} \mathrm{m}^{-3}$ (Fig. 14h) [80].

\subsection{Mechanical Properties of Graphene Fibers}

The first GFs show a $\sigma$ of ca. $140 \mathrm{MPa}$ with breakage elongation of $5.8 \%$, but has good flexibility to make tight knots [46]. Compared to the intrinsic nature of graphene ( $\sigma$, $130 \mathrm{GPa}, E, 1.1 \mathrm{TPa}$ ) [81-83], a plenty of work should be done to improve the mechanical performance of GFs. Based on the subsequent study of GFs, the mechanical performance can be enhanced from three aspects: (1) 
Table 1 Summary of the preparation methods, and mechanical properties of the most relevant CFs

\begin{tabular}{|c|c|c|c|c|c|}
\hline Fiber type & Year & Preparation method & $\begin{array}{l}\text { Strength } \\
(\mathrm{MPa})\end{array}$ & $\begin{array}{l}\text { Modulus } \\
(\mathrm{GPa})\end{array}$ & References \\
\hline \multirow[t]{27}{*}{$\mathrm{CFs}$} & 2000 & Wet-spinning approach, coagulated in PVA solution & 150 & 15 & {$[6]$} \\
\hline & 2002 & Wet-spinning approach, coagulated in PVA solution, stretched & 230 & 40 & {$[92]$} \\
\hline & 2003 & $\begin{array}{l}\text { Wet-spinning approach, SDS surfactant, PVA solution coagulation, post process } \\
\text { with acetone-washing bath }\end{array}$ & 1800 & 80 & [9] \\
\hline & 2004 & Wet-spinning approach, DNA-stabilized dispersions & 109 & 14.3 & [7] \\
\hline & 2004 & Wet-spinning approach, fuming sulfuric acid suspensions & $116 \pm 10$ & $120 \pm 10$ & {$[18]$} \\
\hline & 2004 & Spinning from a CVD reaction zone & $100-1000$ & & {$[28]$} \\
\hline & 2005 & $\begin{array}{l}\text { Wet-spinning approach, polymer-free flocculation-based process, coagulation by } \\
\text { adjust the } \mathrm{pH} \text { of water }\end{array}$ & 770 & 8.9 & [19] \\
\hline & 2005 & Wet-spinning approach, hot-drawing treatments & $1400-1800$ & 45 & {$[11]$} \\
\hline & 2005 & Spun from an aerogel formed by CVD & 1460 & $10-30$ & {$[30]$} \\
\hline & 2006 & Modified coagulation spinning, no surfactant, & 500 & $5-10$ & {$[93]$} \\
\hline & 2006 & Spun from an array of $4.7 \mathrm{~mm} \mathrm{CNT}$ & 3300 & & {$[22]$} \\
\hline & 2007 & Drawing-drying process from CNT cotton & 299 & 8.3 & {$[41]$} \\
\hline & 2007 & Spun from CNT aerogel & 8800 & 397 & {$[31]$} \\
\hline & 2007 & Spun from an array of longer nanotubes & & & {$[25]$} \\
\hline & 2007 & Spun from an CNT array, twisted & $1350-3300$ & $100-263$ & {$[23]$} \\
\hline & 2007 & Spun from an CNT array, twisted & 1910 & 330 & {$[24]$} \\
\hline & 2007 & Spun from an CNT cotton & 180 & & {$[24]$} \\
\hline & 2008 & Wet-spinning approach based on lyotropic liquid crystalline phase & $150 \pm 6$ & $69 \pm 41$ & {$[16]$} \\
\hline & 2008 & Wet-spinning approach, DNA as binder & & 1.25 & {$[8]$} \\
\hline & 2008 & Spun from an CNT array & 1240 & & {$[26]$} \\
\hline & 2010 & Spun from CNT array & 1300 & 95 & [76] \\
\hline & 2011 & Spun from CNT array & $2500 \pm 310$ & $120 \pm 23$ & {$[80]$} \\
\hline & 2013 & Wet-spinning CNT liquid crystals & $1000 \pm 200$ & $120 \pm 50$ & [94] \\
\hline & 2014 & Spun from an aerogel formed by CVD & $3760-5530$ & & {$[95]$} \\
\hline & 2015 & Spun from an aerogel formed by CVD & 1000 & & [96] \\
\hline & 2016 & Twist-spun CNT yarns & 2000 & 170 & {$[97]$} \\
\hline & 2017 & Twisting as-grown SWNT film & $3300-3700$ & $30-80$ & [98] \\
\hline \multirow{19}{*}{$\begin{array}{l}\text { Graphene } \\
\text { fibers }\end{array}$} & 2011 & Wet-spinning approaches & 140 & 7.7 & {$[46]$} \\
\hline & 2012 & Wet-spinning approaches & 182 & 8.7 & {$[52]$} \\
\hline & 2012 & One-step dimensionally confined hydrothermal strategy, treated at $800{ }^{\circ} \mathrm{C}$ & 420 & & {$[66]$} \\
\hline & 2012 & Wet-spinning approaches, HPG grafted & 125 & 8.2 & [59] \\
\hline & 2012 & Wet-spinning approaches, CNT, PVA grafted & 580 & 400 & [79] \\
\hline & 2013 & Wet-spinning approaches, GO LC template, HPG crosslink & 652 & 20.9 & {$[60]$} \\
\hline & 2013 & Wet-spinning approaches, large-sized GO & 360 & 12.8 & {$[55]$} \\
\hline & 2013 & Wet-spinning approaches, large-sized GO & 442 & 22.6 & [49] \\
\hline & 2013 & Wet-spinning approaches, PVA grafted & 199 & 17.1 & {$[61]$} \\
\hline & 2013 & Wet-spinning approaches, PAN grafted & 452 & 8.31 & {$[57]$} \\
\hline & 2013 & Wet-spinning approaches, large-sized GO & 214 & 47 & {$[86]$} \\
\hline & 2013 & Wet-spinning approaches, large-sized $\mathrm{GO}, \mathrm{Ca}^{2+}$ crosslink & 501 & 11.2 & [87] \\
\hline & 2013 & Wet-spinning approaches, PGMA grafted & 500 & 18.8 & {$[58]$} \\
\hline & 2014 & Coaxial wet-spinning approaches & 116 & & {$[56]$} \\
\hline & 2014 & One-step dimensionally confined hydrothermal strategy, vitamin $\mathrm{C}$ reduced & 150 & 1.9 & {$[68]$} \\
\hline & 2015 & Wet-spinning approaches, montmorillonite & 270 & 44 & {$[65]$} \\
\hline & 2015 & Wet-spinning approaches, 30\% small-sized GO, high-temperature graphitization & 1150 & 135 & {$[88]$} \\
\hline & 2016 & Wet-spinning approaches, SA & 784.9 & 58 & {$[62]$} \\
\hline & 2016 & Wet-spinning approaches, high-temperature graphitization & 1450 & 282 & [89] \\
\hline
\end{tabular}




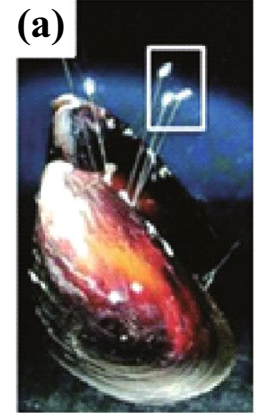

(b)

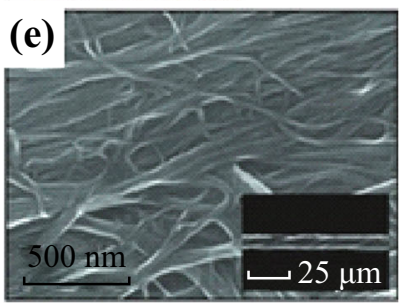

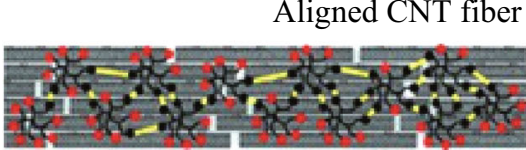

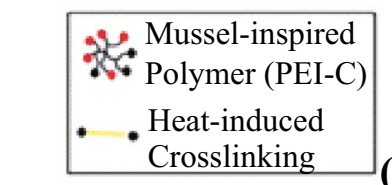

(f)

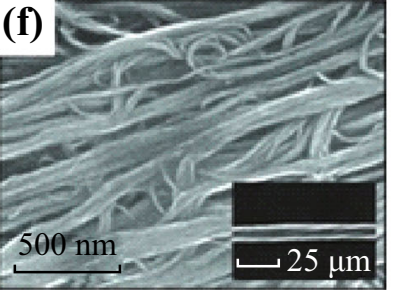

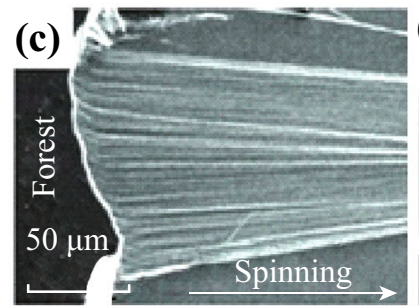
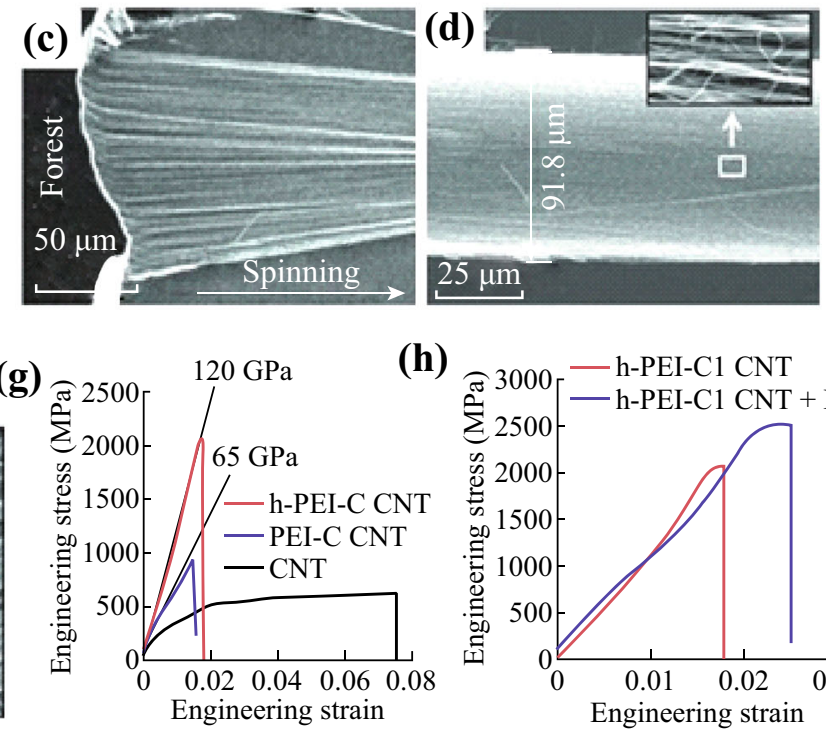

(h)

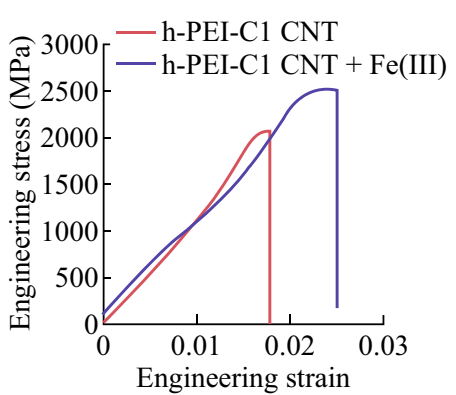

Fig. 14 a Photograph of a mussel attached to Si substrate. b A schematic illustration of one-dimensionally aligned CFs reinforced by musselmimetic adhesives. SEM observations of fiber spinning process $\mathbf{c}$ and the spun CFs (d). High-magnification SEM images of the densified CF e and h-PEI-C treated CF f. Mechanical curves of the CFs. (g, h) Stress-strain curves of h-PEI-C-CNT (red) and h-PEI-C-CNT + Fe(III) (blue). Reproduced with permission from Ref. [80]. Copyright 2011, Wiley-VCH. (Color figure online)

improving the alignment of graphene sheets along the fiber axis; (2) decreasing the structural defects that include sheets boundaries, voids and impurities; and (3) enhancing the interlayer interaction of the constituent graphene sheets by either covalent or noncovalent bonds [84, 85]. The stretching operation leads to a high alignment of GO sheets and benefit for the formation of compact structures [86]. When draw ratios were increased from 1.09 to 1.45 , the $\sigma$ is raised from 124 to $214 \mathrm{MPa}$ and $E$ reaches 47 from $14 \mathrm{GPa}$. To decreasing the structural defects, large-sized GO were used as building block [49, 55, 86, 87]. As a result, the improved $\sigma$ ranged from 214 to $501 \mathrm{MPa}$. To enhance the interlayer interaction, PVA [61], HPG [59], PAN [57], PGMA [58], and SA [62] were introduced into interlayer galleries. The resultant fibers exhibited $\sigma$ of 199 , 652,452 , and $500 \mathrm{MPa}, E$ of $17.1,20.9,8.31$, and $18.8 \mathrm{GPa}$, respectively (as shown in Table 1).

High-temperature graphitization process is a more effective method to achieve high-performance GFs since the structural defects were removed or restored. The $\sigma$ break through $1 \mathrm{GPa}$ for the first time. In 2015, Lian's group improved the mechanical performance of GFs to $1.15 \mathrm{GPa}$ by combining the large-sized graphene with small-sized graphene, aided by flowing-stretching and high-temperature graphitization treatment [88]. Recently, $\mathrm{Xu}$ et al. raised this value to $2.2 \mathrm{GPa}$ through a full-scale synergetic defect engineering method to minimize the possible defects at all levels ranging from atomic to macroscale, the best mechanical performance until now [89-91].

\section{Concluding Remarks and Prospects}

Many methods are developed to assemble CNTs and graphene into macroscopic fibers. For CNT fibers, on account of the entanglement nature between individual nanotubes, the approach of drawing CNT out from superaligned CNT arrays or aerogels is promising in achieved continuous, high-strength and high-electrical-conductive fibers. For GFs, considering the excellent dispersity of GO in water and organic solvent, liquid-state spinning approaches may be more competitive in the future.

Although great progress has been made to improve the mechanical performance of macroscopic CFs and GFs, a wide gap still exists considering the intrinsic properties of CNT and graphene building blocks. Until now, the $\sigma$ of $\mathrm{CFs}$ and GFs both exceed $1 \mathrm{GPa}$, comparable to that of the universal carbon fibers. In order to improve the performance of macroscopic CFs and GFs, defects such as voids and disordered orientation should be minimized.

To further upgrade the combined performance of $\mathrm{CFs}$ and GFs, especially the mechanical strength and electrical conductivity, much work is waiting to be explored and more sophisticated understandings about the relationship between the structure and performance is of significant important from the viewpoints of both fundamental research and real application. On the one hand, we should establish a real-time system to monitor the structural evolution of the basic CNTs/graphene units during the fiberforming process, which will contribute to the structure controlling of the fibers. On the other hand, the processing 
techniques (e.g., stretching, fining, densifying, annealing) used to enhance the performance of polymeric fibers and carbon fibers should be borrowed to optimize the fabrication process throughout.

Acknowledgements This work is supported by the National Natural Science Foundation of China (Nos. 21325417, 51533008), the MOST National Key Research and Development Plan (2016YFA0200200), the National Postdoctoral Program for Innovative Talents (No. BX201700209), and the State Key Laboratory for Modification of Chemical Fibers and Polymer Materials, Donghua University (No. LK1403).

Open Access This article is distributed under the terms of the Creative Commons Attribution 4.0 International License (http://creative commons.org/licenses/by/4.0/), which permits unrestricted use, distribution, and reproduction in any medium, provided you give appropriate credit to the original author(s) and the source, provide a link to the Creative Commons license, and indicate if changes were made.

\section{References}

1. S. Nardecchia, D. Carriazo, M.L. Ferrer, M.C. Gutierrez, F. Del Monte, Three dimensional macroporous architectures and aerogels built of carbon nanotubes and/or graphene: synthesis and applications. Chem. Soc. Rev. 42(2), 794-830 (2013). doi:10. 1039/C2CS35353A

2. Z. Li, Z. Liu, H.Y. Sun, C. Gao, Superstructured assembly of nanocarbons: fullerenes, nanotubes, and graphene. Chem. Rev. 115(15), 7046-7117 (2015). doi:10.1021/acs.chemrev.5b00102

3. L. Liu, W. Ma, Z. Zhang, Macroscopic carbon nanotube assemblies: preparation, properties, and potential applications. Small 7(11), 1504-1520 (2011). doi:10.1002/smll.201002198

4. H.-P. Cong, J.-F. Chen, S.-H. Yu, Graphene-based macroscopic assemblies and architectures: an emerging material system. Chem. Soc. Rev. 43(21), 7295-7325 (2014). doi:10.1039/ $\mathrm{C} 4 \mathrm{CS} 00181 \mathrm{H}$

5. N. Behabtu, M.J. Green, M. Pasquali, Carbon nanotube-based neat fibers. Nano Today 3(5-6), 24-34 (2008). doi:10.1016/ s1748-0132(08)70062-8

6. B. Vigolo, A. Penicaud, C. Coulon, C. Sauder, R. Pailler, C. Journet, P. Bernier, P. Poulin, Macroscopic fibers and ribbons of oriented carbon nanotubes. Science 290(5495), 1331-1334 (2000). doi:10.1126/science.290.5495.1331

7. J.N. Barisci, M. Tahhan, G.G. Wallace, S. Badaire, T. Vaugien, M. Maugey, P. Poulin, Properties of carbon nanotube fibers spun from DNA-stabilized dispersions. Adv. Funct. Mater. 14(2), 133-138 (2004). doi:10.1002/adfm.200304500

8. S.R. Shin, C.K. Lee, I. So, J.H. Jeon, T.M. Kang et al., DNAwrapped single-walled carbon nanotube hybrid fibers for supercapacitors and artificial muscles. Adv. Mater. 20(3), 466-470 (2008). doi:10.1002/adma.200701102

9. A.B. Dalton, S. Collins, E. Munoz, J.M. Razal, V.H. Ebron, J.P. Ferraris, J.N. Coleman, B.G. Kim, R.H. Baughman, Super-tough carbon-nanotube fibres - these extraordinary composite fibres can be woven into electronic textiles. Nature 423(6941), 703 (2003). doi: $10.1038 / 423703 a$

10. E. Munoz, A.B. Dalton, S. Collins, M. Kozlov, J. Razal et al., Multifunctional carbon nanotube composite fibers. Adv. Eng. Mater. 6(10), 801-804 (2004). doi:10.1002/adem.200400092

11. P. Miaudet, S. Badaire, M. Maugey, A. Derre, V. Pichot, P. Launois, P. Poulin, C. Zakri, Hot-drawing of single and multiwall carbon nanotube fibers for high toughness and alignment. Nano Lett. 5(11), 2212-2215 (2005). doi:10.1021/n1051419w

12. V.A. Davis, L.M. Ericson, A.N.G. Parra-Vasquez, H. Fan, Y. Wang et al., Phase behavior and rheology of SWNTs in superacids. Macromolecules 37(1), 154-160 (2004). doi:10.1021/ma0352328

13. P.K. Rai, R.A. Pinnick, A.N.G. Parra-Vasquez, V.A. Davis, H.K. Schmidt, R.H. Hauge, R.E. Smalley, M. Pasquali, Isotropic-nematic phase transition of single-walled carbon nanotubes in strong acids. J. Am. Chem. Soc. 128(2), 591-595 (2006). doi:10. $1021 / \mathrm{ja} 055847 \mathrm{f}$

14. S.J. Zhang, I.A. Kinloch, A.H. Windle, Mesogenicity drives fractionation in lyotropic aqueous suspensions of multiwall carbon nanotubes. Nano Lett. 6(3), 568-572 (2006). doi:10.1021/ n10521322

15. S.J. Zhang, S. Kumar, Carbon nanotubes as liquid crystals. Small 4(9), 1270-1283 (2008). doi:10.1002/smll.200700082

16. S.J. Zhang, K.K.K. Koziol, I.A. Kinloch, A.H. Windle, Macroscopic fibers of well-aligned carbon nanotubes by wet spinning. Small 4(8), 1217-1222 (2008). doi:10.1002/smll.200700998

17. W. Zhou, J. Vavro, C. Guthy, K.I. Winey, J.E. Fischer et al., Single wall carbon nanotube fibers extruded from super-acid suspensions: preferred orientation, electrical, and thermal transport. J. Appl. Phys. 95(2), 649-655 (2004). doi:10.1063/1.1627457

18. L.M. Ericson, H. Fan, H.Q. Peng, V.A. Davis, W. Zhou et al., Macroscopic, neat, single-walled carbon nanotube fibers. Science 305(5689), 1447-1450 (2004). doi:10.1126/science.1101398

19. M.E. Kozlov, R.C. Capps, W.M. Sampson, V.H. Ebron, J.P. Ferraris, R.H. Baughman, Spinning solid and hollow polymerfree carbon nanotube fibers. Adv. Mater. 17(5), 614-617 (2005). doi:10.1002/adma.200401130

20. E.Y. Jang, T.J. Kang, H. Im, S.J. Baek, S. Kim, D.H. Jeong, Y.W. Park, Y.H. Kim, Macroscopic single-walled-carbon-nanotube fiber self-assembled by dip-coating method. Adv. Mater. 21(43), 4357-4361 (2009). doi:10.1002/adma.200900480

21. K. Jiang, Q. Li, S. Fan, Nanotechnology: spinning continuous carbon nanotube yarns. Nature 419(6909), 801 (2002). doi:10. 1038/419801a

22. Q.W. Li, X.F. Zhang, R.F. DePaula, L.X. Zheng, Y.H. Zhao et al., Sustained growth of ultralong carbon nanotube arrays for fiber spinning. Adv. Mater. 18(23), 3160-3163 (2006). doi:10. 1002/adma.200601344

23. X.F. Zhang, Q.W. Li, T.G. Holesinger, P.N. Arendt, J.Y. Huang et al., Ultrastrong, stiff, and lightweight carbon-nanotube fibers. Adv. Mater. 19(23), 4198-4201 (2007). doi:10.1002/adma. 200700776

24. X.F. Zhang, Q.W. Li, Y. Tu, Y.A. Li, J.Y. Coulter et al., Strong carbon-nanotube fibers spun from long carbon-nanotube arrays. Small 3(2), 244-248 (2007). doi:10.1002/smll.200600368

25. Q.W. Li, Y. Li, X.F. Zhang, S.B. Chikkannanavar, Y.H. Zhao et al., Structure-dependent electrical properties of carbon nanotube fibers. Adv. Mater. 19(20), 3358-3363 (2007). doi:10.1002/ adma.200602966

26. H.S. Peng, M. Jain, Q.W. Li, D.E. Peterson, Y.T. Zhu, Q.X. Jia, Vertically aligned pearl-like carbon nanotube arrays for fiber spinning. J. Am. Chem. Soc. 130(4), 1130-1131 (2008). doi:10. $1021 / \mathrm{ja} 077767 \mathrm{c}$

27. H.W. Zhu, C.L. Xu, D.H. Wu, B.Q. Wei, R. Vajtai, P.M. Ajayan, Direct synthesis of long single-walled carbon nanotube strands. Science 296(5569), 884-886 (2002). doi:10.1126/science. 1066996

28. Y.L. Li, I.A. Kinloch, A.H. Windle, Direct spinning of carbon nanotube fibers from chemical vapor deposition synthesis. Science 304(5668), 276-278 (2004). doi:10.1126/science.1094982

29. J.J. Vilatela, A.H. Windle, Yarn-like carbon nanotube fibers. Adv. Mater. 22(44), 4959-4963 (2010). doi:10.1002/adma. 201002131 
30. M. Motta, Y.L. Li, I. Kinloch, A. Windle, Mechanical properties of continuously spun fibers of carbon nanotubes. Nano Lett. 5(8), 1529-1533 (2005). doi:10.1021/n1050634+

31. K. Koziol, J. Vilatela, A. Moisala, M. Motta, P. Cunniff, M. Sennett, A. Windle, High-performance carbon nanotube fiber. Science 318(5858), 1892-1895 (2007). doi:10.1126/science. 1147635

32. X.H. Zhong, Y.L. Li, Y.K. Liu, X.H. Qiao, Y. Feng et al., Continuous multilayered carbon nanotube yarns. Adv. Mater. 22(6), 692-696 (2010). doi:10.1002/adma.200902943

33. W.J. Ma, L.Q. Liu, R. Yang, T.H. Zhang, Z. Zhang et al., Monitoring a micromechanical process in macroscale carbon nanotube films and fibers. Adv. Mater. 21(5), 603-608 (2009). doi:10.1002/adma.200801335

34. J.M. Feng, R. Wang, Y.L. Li, X.H. Zhong, L. Cui, Q.J. Guo, F. Hou, One-step fabrication of high quality double-walled carbon nanotube thin films by a chemical vapor deposition process. Carbon 48(13), 3817-3824 (2010). doi:10.1016/j.carbon.2010.06.046

35. W.J. Ma, L.Q. Liu, Z. Zhang, R. Yang, G. Liu et al., Highstrength composite fibers: realizing true potential of carbon nanotubes in polymer matrix through continuous reticulate architecture and molecular level couplings. Nano Lett. 9(8), 2855-2861 (2009). doi:10.1021/n1901035v

36. Y. Shang, X. He, Y. Li, L. Zhang, Z. Li et al., Super-stretchable spring-like carbon nanotube ropes. Adv. Mater. 24(21), 2896-2900 (2012). doi:10.1002/adma.201200576

37. Y.Y. Shang, Y.B. Li, X.D. He, L.H. Zhang, Z. Li, P.X. Li, E.Z. Shi, S.T. Wu, A.Y. Cao, Elastic carbon nanotube straight yarns embedded with helical loops. Nanoscale 5(6), 2403-2410 (2013). doi:10.1039/c3nr33633f

38. Y.B. Li, Y.Y. Shang, X.D. He, Q.Y. Peng, S.Y. Du et al., Overtwisted, resolvable carbon nanotube yarn entanglement as strain sensors and rotational actuators. ACS Nano 7(9), 8128-8135 (2013). doi:10.1021/nn403400c

39. Y.Y. Shang, Y.B. Li, X.D. He, S.Y. Du, L.H. Zhang et al., Highly twisted double-helix carbon nanotube yarns. ACS Nano 7(2), 1446-1453 (2013). doi:10.1021/nn305209h

40. M.D. Lima, S.L. Fang, X. Lepro, C. Lewis, R. Ovalle-Robles et al., Biscrolling nanotube sheets and functional guests into yarns. Science 331(6013), 51-55 (2011). doi:10.1126/science. 1195912

41. L. Ci, N. Punbusayakul, J.Q. Wei, R. Vajtai, S. Talapatra, P.M. Ajayan, Multifunctional macroarchitectures of double-walled carbon nanotube fibers. Adv. Mater. 19(13), 1719-1723 (2007). doi:10.1002/adma.200602520

42. L.X. Zheng, X.F. Zhang, Q.W. Li, S.B. Chikkannanavar, Y. Li et al., Carbon-nanotube cotton for large-scale fibers. Adv. Mater. 19(18), 2567-2570 (2007). doi:10.1002/adma.200602648

43. H.H. Gommans, J.W. Alldredge, H. Tashiro, J. Park, J. Magnuson, A.G. Rinzler, Fibers of aligned single-walled carbon nanotubes: polarized Raman spectroscopy. J. Appl. Phys. 88(5), 2509-2514 (2000). doi:10.1063/1.1287128

44. G.T. Liu, Y.C. Zhao, K. Deng, Z. Liu, W.G. Chu et al., Highly dense and perfectly aligned single-walled carbon nanotubes fabricated by diamond wire drawing dies. Nano Lett. 8(4), 1071-1075 (2008). doi:10.1021/n1073007o

45. Z. Liu, K.H. Zheng, L.J. Hu, J. Liu, C.Y. Qiu et al., Surfaceenergy generator of single-walled carbon nanotubes and usage in a self-powered system. Adv. Mater. 22(9), 999-1003 (2010). doi:10.1002/adma.200902153

46. Z. Xu, C. Gao, Graphene chiral liquid crystals and macroscopic assembled fibres. Nat. Commun. 2, 571 (2011). doi:10.1038/ ncomms 1583

47. Z. Xu, C. Gao, Aqueous liquid crystals of graphene oxide. ACS Nano 5(4), 2908-2915 (2011). doi:10.1021/nn200069w
48. N. Behabtu, J.R. Lomeda, M.J. Green, A.L. Higginbotham, A. Sinitskii et al., Spontaneous high-concentration dispersions and liquid crystals of graphene. Nat. Nanotechnol. 5(6), 406-411 (2010). doi:10.1038/nnano.2010.86

49. R. Jalili, S.H. Aboutalebi, D. Esrafilzadeh, R.L. Shepherd, J. Chen et al., Scalable one-step wet-spinning of graphene fibers and yarns from liquid crystalline dispersions of graphene oxide: towards multifunctional textiles. Adv. Funct. Mater. 23(43), 5345-5354 (2013). doi:10.1002/adfm.201300765

50. B. Zheng, T. Huang, L. Kou, X. Zhao, K. Gopalsamy, C. Gao, Graphene fiber-based asymmetric micro-supercapacitors. J. Mater. Chem. A 2(25), 9736-9743 (2014). doi:10.1039/C4TA01868K

51. T.Q. Huang, B.N. Zheng, L. Kou, K. Gopalsamy, Z. Xu, C. Gao, Y.N. Meng, Z.X. Wei, Flexible high performance wet-spun graphene fiber supercapacitors. RSC Adv. 3(46), 23957-23962 (2013). doi:10.1039/c3ra44935a

52. H.P. Cong, X.C. Ren, P. Wang, S.H. Yu, Wet-spinning assembly of continuous, neat, and macroscopic graphene fibers. Sci. Rep. 2 , 613 (2012). doi:10.1038/srep00613

53. J. Sun, Y. Li, Q. Peng, S. Hou, D. Zou et al., Macroscopic, flexible, high-performance graphene ribbons. ACS Nano 7(11), 10225-10232 (2013). doi:10.1021/nn404533r

54. Z. Xu, Y. Zhang, P. Li, C. Gao, Strong, conductive, lightweight, neat graphene aerogel fibers with aligned pores. ACS Nano 6(8), 7103-7113 (2012). doi:10.1021/nn3021772

55. Y. Zhao, C.C. Jiang, C.G. Hu, Z.L. Dong, J.L. Xue, Y.N. Meng, N. Zheng, P.W. Chen, L.T. Qu, Large-scale spinning assembly of neat, morphology-defined, graphene-based hollow fibers. ACS Nano 7(3), 2406-2412 (2013). doi:10.1021/nn305674a

56. L. Kou, T. Huang, B. Zheng, Y. Han, X. Zhao, K. Gopalsamy, H. Sun, C. Gao, Coaxial wet-spun yarn supercapacitors for highenergy density and safe wearable electronics. Nat. Commun. 5, 3754 (2014). doi: $10.1038 /$ ncomms 4754

57. Z. Liu, Z. Xu, X.Z. Hu, C. Gao, Lyotropic liquid crystal of polyacrylonitrile-grafted graphene oxide and its assembled continuous strong nacre-mimetic fibers. Macromolecules 46(17), 6931-6941 (2013). doi:10.1021/ma400681v

58. X.L. Zhao, Z. Xu, B.N. Zheng, C. Gao, Macroscopic assembled, ultrastrong and $\mathrm{H}_{2} \mathrm{SO}_{4}$-resistant fibres of polymer-grafted graphene oxide. Sci. Rep. 3, 3164 (2013). doi:10.1038/srep03164

59. X.Z. Hu, Z. Xu, C. Gao, Multifunctional, supramolecular, continuous artificial nacre fibres. Sci. Rep. 2, 767 (2012). doi:10. 1038/srep00767

60. X.Z. Hu, Z. Xu, Z. Liu, C. Gao, Liquid crystal self-templating approach to ultrastrong and tough biomimic composites. Sci. Rep. 3, 2374 (2013). doi:10.1038/srep02374

61. L. Kou, C. Gao, Bioinspired design and macroscopic assembly of poly(vinyl alcohol)-coated graphene into kilometers-long fibers. Nanoscale 5(10), 4370-4378 (2013). doi:10.1039/c3nr00455d

62. X. Hu, S. Rajendran, Y. Yao, Z. Liu, K. Gopalsamy, L. Peng, C. Gao, A novel wet-spinning method of manufacturing continuous bio-inspired composites based on graphene oxide and sodium alginate. Nano Res. 9(3), 735-744 (2016). doi:10.1007/s12274015-0952-2

63. Z. Xu, Z. Liu, H.Y. Sun, C. Gao, Highly electrically conductive Ag-doped graphene fibers as stretchable conductors. Adv. Mater. 25(23), 3249-3253 (2013). doi:10.1002/adma.201300774

64. K. Gopalsamy, Z. Xu, B. Zheng, T. Huang, L. Kou, X. Zhao, C. Gao, Bismuth oxide nanotubes-graphene fiber-based flexible supercapacitors. Nanoscale 6(15), 8595-8600 (2014). doi:10. 1039/C4NR02615B

65. B. Fang, L. Peng, Z. Xu, C. Gao, Wet-spinning of continuous montmorillonite-graphene fibers for fire-resistant lightweight conductors. ACS Nano 9(5), 5214-5222 (2015). doi:10.1021/ acsnano. 5 b00616 
66. Z.L. Dong, C.C. Jiang, H.H. Cheng, Y. Zhao, G.Q. Shi, L. Jiang, L.T. Qu, Facile fabrication of light, flexible and multifunctional graphene fibers. Adv. Mater. 24(14), 1856-1861 (2012). doi:10. 1002/adma.201200170

67. H.H. Cheng, Z.L. Dong, C.G. Hu, Y. Zhao, Y. Hu, L.T. Qu, N. Chena, L.M. Dai, Textile electrodes woven by carbon nanotubegraphene hybrid fibers for flexible electrochemical capacitors. Nanoscale 5(8), 3428-3434 (2013). doi:10.1039/c3nr00320e

68. J. Li, J. Li, L. Li, M. Yu, H. Ma, B. Zhang, Flexible graphene fibers prepared by chemical reduction-induced self-assembly. J. Mater. Chem. A 2(18), 6359-6362 (2014). doi:10.1039/ C4TA00431K

69. E.Y. Jang, J. Carretero-Gonzalez, A. Choi, W.J. Kim, M.E. Kozlov et al., Fibers of reduced graphene oxide nanoribbons. Nanotechnology 23(23), 235601-235608 (2012). doi:10.1088/ 0957-4484/23/23/235601

70. X.M. Li, T.S. Zhao, K.L. Wang, Y. Yang, J.Q. Wei, F.Y. Kang, D.H. Wu, H.W. Zhu, Directly drawing self-assembled, porous, and monolithic graphene fiber from chemical vapor deposition grown graphene film and its electrochemical properties. Langmuir 27(19), 12164-12171 (2011). doi:10.1021/la202380g

71. X.M. Li, T.S. Zhao, Q. Chen, P.X. Li, K.L. Wang et al., Flexible all solid-state supercapacitors based on chemical vapor deposition derived graphene fibers. Phys. Chem. Chem. Phys. 15(41), 17752-17757 (2013). doi:10.1039/c3cp52908h

72. D.D. Nguyen, S. Suzuki, S. Kato, B.D. To, C.C. Hsu, H. Murata, E. Rokuta, N.-H. Tai, M. Yoshimura, Macroscopic, freestanding, and tubular graphene architectures fabricated via thermal annealing. ACS Nano 9(3), 3206-3214 (2015). doi:10.1021/acs nano.5b00292

73. M. Xiao, T. Kong, W. Wang, Q. Song, D. Zhang, Q. Ma, G. Cheng, Interconnected graphene networks with uniform geometry for flexible conductors. Adv. Funct. Mater. 25(39), 6165-6172 (2015). doi:10.1002/adfm.201502966

74. X. Shao, A. Srinivasan, Y. Zhao, A. Khursheed, A few-layer graphene ring-cathode field emitter for focused electron/ion beam applications. Carbon 110, 378-383 (2016). doi:10.1016/j.carbon. 2016.09.048

75. M. Naraghi, T. Filleter, A. Moravsky, M. Locascio, R.O. Loutfy, H.D. Espinosa, A multiscale study of high performance doublewalled nanotube-polymer fibers. ACS Nano 4(11), 6463-6476 (2010). doi: $10.1021 / \mathrm{nn} 101404 \mathrm{u}$

76. L.X. Zheng, G.Z. Sun, Z.Y. Zhan, Tuning array morphology for high-strength carbon-nanotube fibers. Small 6(1), 132-137 (2010). doi:10.1002/smll.200900954

77. L. Kai, S. Yinghui, Z. Ruifeng, Z. Hanyu, W. Jiaping, L. Liang, F. Shoushan, J. Kaili, Carbon nanotube yarns with high tensile strength made by a twisting and shrinking method. Nanotechnology 21(4), 045708 (2010). doi:10.1088/0957-4484/21/4/ 045708

78. A. Kis, G. Csanyi, J.P. Salvetat, T.-N. Lee, E. Couteau, A.J. Kulik, W. Benoit, J. Brugger, L. Forro, Reinforcement of singlewalled carbon nanotube bundles by intertube bridging. Nat. Mater. 3(3), 153-157 (2004). doi:10.1038/nmat1076

79. M.K. Shin, B. Lee, S.H. Kim, J.A. Lee, G.M. Spinks et al., Synergistic toughening of composite fibres by self-alignment of reduced graphene oxide and carbon nanotubes. Nat. Commun. 3, 650 (2012). doi:10.1038/ncomms 1661

80. S. Ryu, Y. Lee, J.W. Hwang, S. Hong, C. Kim, T.G. Park, H. Lee, S.H. Hong, High-strength carbon nanotube fibers fabricated by infiltration and curing of mussel-inspired catecholamine polymer. Adv. Mater. 23(17), 1971-1975 (2011). doi:10.1002/adma. 201004228
81. K.S. Novoselov, A.K. Geim, S.V. Morozov, D. Jiang, Y. Zhang, S.V. Dubonos, I.V. Grigorieva, A.A. Firsov, Electric field effect in atomically thin carbon films. Science 306(5696), 666-669 (2004). doi:10.1126/science.1102896

82. A.K. Geim, Graphene: status and prospects. Science 324(5934), 1530-1534 (2009). doi:10.1126/science. 1158877

83. M.J. Allen, V.C. Tung, R.B. Kaner, Honeycomb carbon: a review of graphene. Chem. Rev. 110(1), 132-145 (2010). doi:10.1021/ cr900070d

84. Z. $\mathrm{Xu}, \mathrm{C}$. Gao, Graphene fiber: a new trend in carbon fibers. Mater. Today 18(9), 480-492 (2015). doi:10.1016/j.mattod.2015. 06.009

85. L. Chen, Y. He, S. Chai, H. Qiang, F. Chen, Q. Fu, Toward high performance graphene fibers. Nanoscale 5(13), 5809-5815 (2013). doi:10.1039/C3NR01083J

86. C. Xiang, C.C. Young, X. Wang, Z. Yan, C.-C. Hwang et al., Large flake graphene oxide fibers with unconventional $100 \%$ knot efficiency and highly aligned small flake graphene oxide fibers. Adv. Mater. 25(33), 4592-4597 (2013). doi:10.1002/adma. 201301065

87. Z. Xu, H. Sun, X. Zhao, C. Gao, Ultrastrong fibers assembled from giant graphene oxide sheets. Adv. Mater. 25(2), 188-193 (2013). doi:10.1002/adma.201203448

88. G. Xin, T. Yao, H. Sun, S.M. Scott, D. Shao, G. Wang, J. Lian, Highly thermally conductive and mechanically strong graphene fibers. Science 349(6252), 1083-1087 (2015). doi:10.1126/sci ence.aaa6502

89. Z. Xu, Y. Liu, X. Zhao, L. Peng, H. Sun et al., Ultrastiff and strong graphene fibers via full-scale synergetic defect engineering. Adv. Mater. 28(30), 6449-6456 (2016). doi:10.1002/adma. 201506426

90. Y. Liu, Z. Xu, J. Zhan, P. Li, C. Gao, Superb electrically conductive graphene fibers via doping strategy. Adv. Mater. 28(36), 7941-7947 (2016). doi:10.1002/adma.201602444

91. Y. Liu, Z. Xu, W. Gao, Z. Cheng, C. Gao, Graphene and other 2D colloids: liquid crystals and macroscopic fibers. Adv. Mater. 29(14), 1606794 (2017). doi:10.1002/adma.201606794

92. B. Vigolo, P. Poulin, M. Lucas, P. Launois, P. Bernier, Improved structure and properties of single-wall carbon nanotube spun fibers. Appl. Phys. Lett. 81(7), 1210-1212 (2002). doi:10.1063/1. 1497706

93. W. Neri, M. Maugey, P. Miaudet, A. Derre, C. Zakri, P. Poulin, Surfactant-free spinning of composite carbon nanotube fibers. Macromol. Rapid Commun. 27(13), 1035-1038 (2006). doi:10. 1002/marc.200600150

94. N. Behabtu, C.C. Young, D.E. Tsentalovich, O. Kleinerman, X. Wang et al., Strong, light, multifunctional fibers of carbon nanotubes with ultrahigh conductivity. Science 339(6116), 182-186 (2013). doi:10.1126/science.1228061

95. J.N. Wang, X.G. Luo, T. Wu, Y. Chen, High-strength carbon nanotube fibre-like ribbon with high ductility and high electrical conductivity. Nat. Commun. 5, 3848 (2014). doi:10.1038/ ncomms 4848

96. B. Alemán, V. Reguero, B. Mas, J.J. Vilatela, Strong carbon nanotube fibers by drawing inspiration from polymer fiber spinning. ACS Nano 9(7), 7392-7398 (2015). doi:10.1021/acsnano.5b02408

97. J. Di, S. Fang, F.A. Moura, D.S. Galvão, J. Bykova et al., Strong, twist-stable carbon nanotube yarns and muscles by tension annealing at extreme temperatures. Adv. Mater. 28(31), 6598-6605 (2016). doi:10.1002/adma.201600628

98. Y. Shang, Y. Wang, S. Li, C. Hua, M. Zou, A. Cao, High-strength carbon nanotube fibers by twist-induced self-strengthening. Carbon 119, 47-55 (2017). doi:10.1016/j.carbon.2017.03.101 